\title{
How constructive is constructing measures?
}

\author{
ARNO PAULY \\ WILLEM L. FOUCHÉ
}

\begin{abstract}
Given some set, how hard is it to construct a measure supported by it? We classify some variations of this task in the Weihrauch lattice. Particular attention is paid to Frostman measures on sets with positive Hausdorff dimension. As a side result, the Weihrauch degree of Hausdorff dimension itself is determined.
\end{abstract}

2010 Mathematics Subject Classification 03D78, 03B30, 28A33 (primary)

Keywords: measure theory, computable analysis, Hausdorff dimension, Frostman Lemma, Weihrauch reducibility

\section{Introduction}

We investigate variations on the problem of constructing a measure with a given support. The variations include the available information about the set, whether the support has to be precisely the given set or merely a subset, and whether the measure is required to be non-atomic. A special case of particular importance is the Frostman lemma, which links having certain Hausdorff dimension to admitting a measure with certain properties. For the relevance of having a computable understanding of Frostman's lemma and of its links with algorithmic randomness and Fourier analysis, the reader is referred to Fouché, Mukeru, and Davie [17].

Two of these variations are computable: Given an overt set $A$, one can construct a measure with support precisely $A$ (Theorem 31 ). In a very restricted setting, a computable version of the Frostman lemma is available (Lemma 59). Apart from these cases, the problems generally are non-computable. Using the framework of Weihrauch reducibility, we can establish the precise degree of non-computability of each case.

The study of Weihrauch degrees forms a framework for the research programme aiming at classifying the computational content of mathematical theorems as formulated and developed by Brattka and Gherardi [5] (also Gherardi and Marcone [19] and Pauly [36]). The core idea is that $S$ is Weihrauch reducible to $T$, if $S$ can be solved using a single invocation of $T$ and otherwise computable means. 


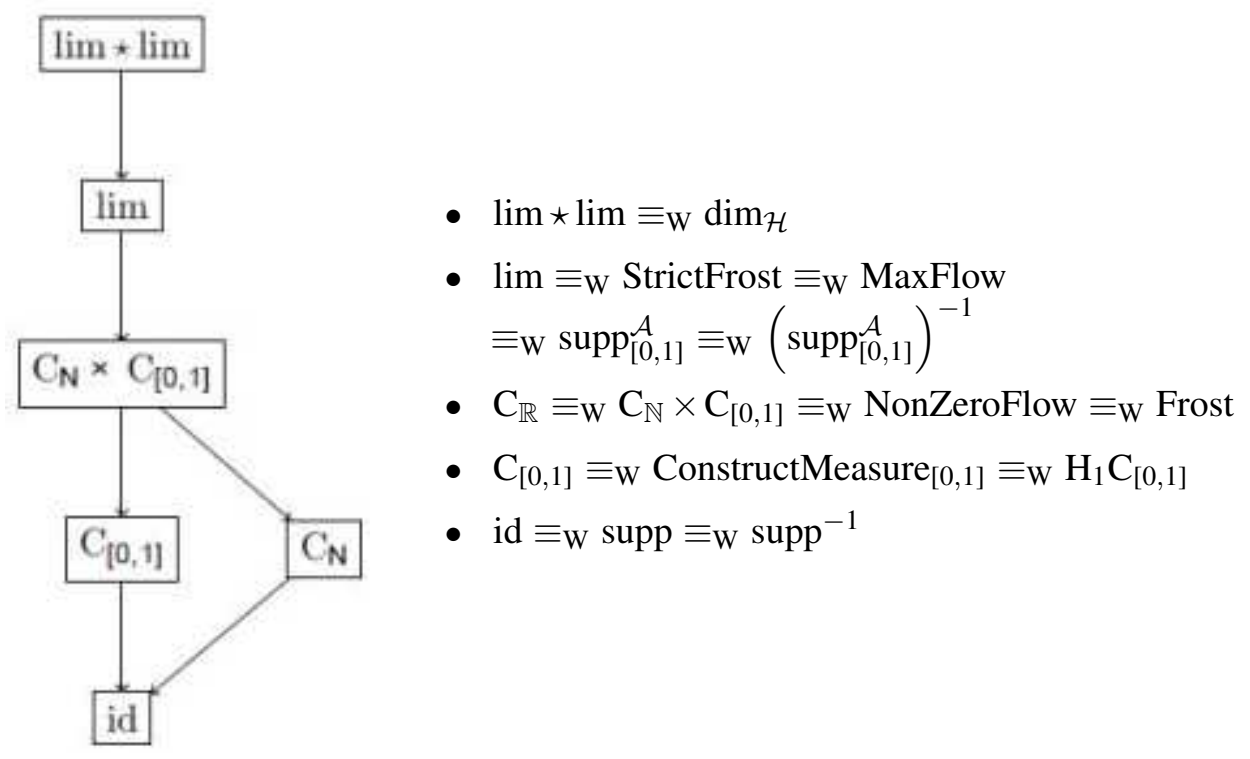

Figure 1: Overview of some the relevant Weihrauch degrees

Numerous theorems have been classified so far. Some examples are the separable Hahn-Banach theorem (Gherardi and Marcone [19]), the Intermediate Value Theorem (Brattka and Gherardi [5]), Nash's theorem for bimatrix games (Pauly [36]), Brouwer's Fixed Point theorem (Brattka, Le Roux and Pauly [10]), the Bolzano-Weierstrass theorem (Brattka, Gherardi and Marcone [8]), the Radon-Nikodym derivative (Hoyrup, Rojas and Weihrauch [26]), the Lebesgue Density Lemma (Brattka, Gherardi and Hölzl [7]), the Goerde-Browder-Kirk fixed point theorem (Neumann [33]) and variants of determinacy of infinite sequential games (Le Roux and Pauly [30, 28]).

An overview of the standard degrees relevant for the classifications in this paper, together with a table which problems classified here fall into which degree, is found in Figure 1.

\section{Background}

\subsection{A short introduction to represented spaces}

We briefly present some fundamental concepts on represented spaces following Pauly [38], to which the reader shall also be referred for a more detailed presentation. The concept behind represented spaces essentially goes back to Weihrauch and 
Kreitz [27], the name may have first been used by Brattka [1]. A represented space is a pair $\mathbf{X}=\left(X, \delta_{X}\right)$ of a set $X$ and a partial surjection $\delta_{X}: \subseteq \mathbb{N}^{\mathbb{N}} \rightarrow X$. A function between represented spaces is a function between the underlying sets. For $f: \subseteq \mathbf{X} \rightarrow \mathbf{Y}$ and $F: \subseteq \mathbb{N}^{\mathbb{N}} \rightarrow \mathbb{N}^{\mathbb{N}}$, we call $F$ a realizer of $f$ (notation $F \vdash f$ ) iff $\delta_{Y}(F(p))=f\left(\delta_{X}(p)\right.$ ) for all $p \in \operatorname{dom}\left(f \delta_{X}\right)$, ie if the following diagram commutes:

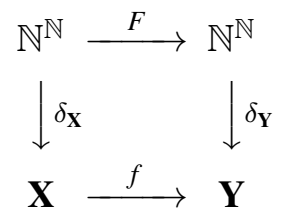

A map between represented spaces is called computable (continuous) iff it has a computable (continuous) realizer. Similarly, we call a point $x \in \mathbf{X}$ computable iff there is some computable $p \in \mathbb{N}^{\mathbb{N}}$ with $\delta_{\mathbf{X}}(p)=x$. A priori, the notion of a continuous map between represented spaces and a continuous map between topological spaces are distinct and should not be confused!

Given two represented spaces $\mathbf{X}, \mathbf{Y}$ we obtain a third represented space $\mathcal{C}(\mathbf{X}, \mathbf{Y})$ of functions from $X$ to $Y$ by letting $0^{n} 1 p$ be a $\left[\delta_{X} \rightarrow \delta_{Y}\right]$-name for $f$, if the $n$-th Turing machine equipped with the oracle $p$ computes a realizer for $f$. The reader should not be mislead by the explicit use of Turing machines in the definition of $\mathcal{C}(\mathbf{X}, \mathbf{Y})$; this is the natural construction of the space of continuous functions. Formally, $\mathcal{C}(-,-)$ is the exponential in the category of continuous maps between represented spaces (as a consequence of the UTM theorem), and the evaluation map is even computable (as are the other canonic maps, eg currying).

This function space constructor, together with two represented spaces, $\mathbb{N}=\left(\mathbb{N}, \delta_{\mathbb{N}}\right)$ (which we understand to include 0$)$ and $\mathbb{S}=\left(\{\perp, \top\}, \delta_{\mathbb{S}}\right)$, allows us to obtain a model of Escardó's synthetic topology [15]. The representations are given by $\delta_{\mathbb{N}}\left(0^{n} 10^{\mathbb{N}}\right)=n$, $\delta_{\mathbb{S}}\left(0^{\mathbb{N}}\right)=\perp$ and $\delta_{\mathbb{S}}(p)=\top$ for $p \neq 0^{\mathbb{N}}$. It is straightforward to verify that the computability notion for the represented space $\mathbb{N}$ coincides with classical computability over the natural numbers. The Sierpiński space $\mathbb{S}$ in turn allows us to formalize semi-decidability. The computable functions $f: \mathbb{N} \rightarrow \mathbb{S}$ are exactly those where $f^{-1}(\{\top\})$ is recursively enumerable (and thus $f^{-1}(\{\perp\})$ co-recursively enumerable).

In general, for any represented space $\mathbf{X}$ we obtain two spaces of subsets of $\mathbf{X}$; the space of open sets $\mathcal{O}(\mathbf{X})$ by identifying $f \in \mathcal{C}(\mathbf{X}, \mathbb{S})$ with $f^{-1}(\{\top\})$. Note that the elements of $\mathcal{O}(\mathbf{X})$ are precisely the open sets in the final topology along $\delta_{\mathbf{X}}$. As a dual notion, we obtain the space of closed $^{1}$ sets $\mathcal{A}(\mathbf{X})$ by identifying $f \in \mathcal{C}(\mathbf{X}, \mathbb{S})$ with $f^{-1}(\{\perp\})$. The properties of the spaces of open and closed sets, namely computability

\footnotetext{
${ }^{1}$ There is a very unfortunate confusing nomenclature here. The computable closed sets in
} 
of the usual operations, follow from computability of the functions $\wedge, \vee: \mathbb{S} \times \mathbb{S} \rightarrow \mathbb{S}$ and $\bigvee: \mathcal{C}(\mathbb{N}, \mathbb{S}) \rightarrow \mathbb{S}$

One useful consequence of staying within the category when forming the space $\mathcal{O}(\mathbf{X})$ is that we can iterate this to obtain $\mathcal{O}(\mathcal{O}(\mathbf{X}))$, a space appearing in several further constructions. We introduce the space $\mathcal{K}(\mathbf{X})$ of compact sets by identifying 2 a set $A \subseteq \mathbf{X}$ with $\{U \in \mathcal{O}(\mathbf{X}) \mid A \subseteq U\} \in \mathcal{O}(\mathcal{O}(\mathbf{X}))$, and define a set $A$ to be compact iff $\{U \in \mathcal{O}(\mathbf{X}) \mid A \subseteq U\}$ is open. For computable metric spaces (introduced below), this notion of compactness coincides with the familiar one. For more details, see Pauly [38, Section 5]. A space $\mathbf{X}$ is called computably compact if $X$ is a computable element of $\mathcal{K}(\mathbf{X})$.

As a dual notion, we find the space of overt set $\mathcal{V}(\mathbf{X})$ by identifying $A \subseteq \mathbf{X}$ with $\{U \in \mathcal{O}(\mathbf{X}) \mid A \cap U \neq \emptyset\} \in \mathcal{O}(\mathcal{O}(\mathbf{X}))$. As this identification erases the distinction between a set and its closure, we will assume all $A \in \mathcal{V}(\mathbf{X})$ to be closed sets. Note however that the properties of $\mathcal{V}(\mathbf{X})$ are very different from $\mathcal{A}(\mathbf{X})$, and thus closed sets and overt sets need to be distinguished (cf [38, Section 7]).

Next, we will recall the operation $\wedge$ on represented spaces. Given two spaces $\mathbf{X}=\left(X, \delta_{\mathbf{X}}\right)$ and $\mathbf{Y}=\left(Y, \delta_{\mathbf{Y}}\right)$, the underlying set of $\mathbf{X} \wedge \mathbf{Y}$ is $X \cap Y$, and the representation $\delta_{\wedge}$ is given by $\delta_{\wedge}(\langle p, q\rangle)=x$ iff $\delta_{\mathbf{X}}(p)=x \wedge \delta_{\mathbf{Y}}(q)=x$. This construction is used to introduce the space $\mathcal{A}(\mathbf{X}) \wedge \mathcal{V}(\mathbf{X})$ of closed and overt subsets.

There always is a canonic computable map $\kappa_{\mathbf{X}}: \mathbf{X} \rightarrow \mathcal{O}(\mathcal{O}(\mathbf{X}))$ defined via $\kappa_{\mathbf{X}}(x)=$ $\{U \mid x \in U\}$. Using the spaces introduced above, we can read the type of $\kappa_{\mathbf{X}}$ alternatively as $\kappa_{\mathbf{X}}: \mathbf{X} \rightarrow \mathcal{K}(\mathbf{X})$ or $\kappa_{\mathbf{X}}: \mathbf{X} \rightarrow \mathcal{V}(\mathbf{X})$ instead. The image of $\mathbf{X}$ under $\kappa_{\mathbf{X}}$ shall be denoted by $\mathbf{X}_{\kappa}$. The following definition essentially goes back to Schröder [41] and provides an effective counterpart to the definition in Schröder [42]:

Definition 1 A space $\mathbf{X}$ is called computably admissible if $\mathbf{X}$ and $\mathbf{X}_{\kappa}$ are computably isomorphic.

Note that $\mathbf{X}_{\kappa}$ is always computably admissible, ie isomorphic to $\left(\mathbf{X}_{\kappa}\right)_{\kappa}$. The computably admissible spaces are precisely those that can be regarded as topological spaces, based on the fact that the computable map $f \mapsto f^{-1}: \mathcal{C}(\mathbf{X}, \mathbf{Y}) \rightarrow \mathcal{C}(\mathcal{O}(\mathbf{Y}), \mathcal{O}(\mathbf{X}))$ becomes computably invertible iff $\mathbf{Y}$ is computably admissible. In other words, the subcategory

our terminology are often called co-c.e. closed following Weihrauch. The sets Weihrauch calls computably closed are the computable closed and overt sets introduced below.

${ }^{2}$ To be precise, this construction gives us only the saturated compact sets. As in a $T_{1}$ space every set is saturated, and we only use $\mathcal{K}(\mathbf{X})$ for such spaces, we gloss over this distinction in the following. 
of admissibly represented spaces is isomorphic to a subcategory of the sequential topological spaces (the $\mathrm{QCB}_{0}$-spaces). For details, see [42] or [38, Section 9].

As a special case of represented spaces, we define computable metric spaces following Weihrauch [47]. The computable Polish spaces are derived from complete computable metric spaces by forgetting the details of the metric, and just retaining the representation (or rather, the equivalence class of representations under computable translations).

Definition 2 We define a computable metric space with its Cauchy representation such that:

(1) A computable metric space is a tuple $\mathbf{M}=\left(M, d,\left(a_{n}\right)_{n \in \mathbb{N}}\right)$ such that $(M, d)$ is a metric space and $\left(a_{n}\right)_{n \in \mathbb{N}}$ is a dense sequence in $(M, d)$.

(2) The relation

$$
\left\{(t, u, v, w) \mid 2^{-t}<d\left(a_{u}, a_{v}\right)<2^{-w}\right\} \text { is recursively enumerable. }
$$

(3) The Cauchy representation $\delta_{\mathbf{M}}: \subseteq \mathbb{N}^{\mathbb{N}} \rightarrow M$ associated with the computable metric space $\mathbf{M}=\left(M, d,\left(a_{n}\right)_{n \in \mathbb{N}}\right)$ is defined by

$$
\delta_{\mathbf{M}}(p)=x: \Longleftrightarrow\left\{\begin{array}{l}
d\left(a_{p(i)}, a_{p(k)}\right) \leq 2^{-i} \text { for } i<k \\
\text { and } x=\lim _{i \rightarrow \infty} a_{p(i)}
\end{array}\right.
$$

The following well-known characterization of the open sets in computable metric spaces is useful for us:

Proposition 3 (Special case of Gregoriades, Kispéter, and Pauly [21, Proposition 13]) Let $\mathbf{M}=\left(M, d,\left(a_{n}\right)_{n \in \mathbb{N}}\right)$ be a computable metric spaces, and a basis $\left(U_{i}\right)_{i \in \mathbb{N}}$ be given via $U_{\langle i, k\rangle}=B\left(a_{i}, 2^{-k}\right)$. Then the map $\bigcup: \mathcal{O}(\mathbb{N}) \rightarrow \mathcal{O}(\mathbf{X})$ defined via $\bigcup(S)=\bigcup_{i \in S} U_{i}$ is computable and has a computable multivalued inverse.

In a computable Polish space, we also have the following very useful characterization of overt sets:

Proposition 4 (Brattka and Presser [13]) Let $\mathbf{X}$ be a complete computable metric space. Then the map $\left(x_{i}\right)_{i \in \mathbb{N}} \mapsto \overline{\left\{x_{i} \mid i \in \mathbb{N}\right\}}: \mathcal{C}(\mathbb{N}, \mathbf{X}) \rightarrow \mathcal{K}(\mathbf{X})$ is computable and has a computable inverse.

Corollary 5 The map OvertChoice $: \subseteq \mathcal{V}(\mathbf{M}) \rightrightarrows \mathbf{M}$ where $x \in$ OvertChoice $(A)$ iff $x \in A$ is computable for any computable Polish space $\mathbf{M}$. 


\subsection{Computable measure theory}

We give a very brief introduction to computable measure theory, to the extent required for our purposes. A more detailed (and very elegant) introduction to the subject can be found in [14] by Collins. We use further special represented space for its development: We can introduce the space $\mathbb{R}_{<}$by identifying a real number $x$ with the set $\{y \in \mathbb{R} \mid y<x\} \in \mathcal{O}(\mathbb{R})$. Equivalently, using $\{y \in \mathbb{Q} \mid y<x\} \in \mathcal{O}(\mathbb{Q})$ provides the same result. A third way is to use a monotone growing sequence $\left(q_{n}\right)_{n \in \mathbb{N}} \in \mathcal{C}(\mathbb{N}, \mathbb{Q})$ as a stand-in for $\sup _{n \in \mathbb{N}} q_{n} \in \mathbb{R}$. We will make use of the following:

Lemma $6 U \mapsto\left\{y \in \mathbb{R} \mid \exists\left(x_{0}, x_{1}, \ldots\right) \in U\left(\sum_{i \in \mathbb{N}} x_{i} \leq y\right)\right\}: \mathcal{O}\left([0,1]^{\mathbb{N}}\right) \rightarrow \mathcal{O}\left(\mathbb{R}_{<}\right)$ is computable.

Proof Using type conversion, we show instead that given $U \in \mathcal{O}\left([0,1]^{\mathbb{N}}\right)$ and $y \in \mathbb{R}$, it is recognizable if $\exists\left(x_{0}, x_{1}, \ldots\right) \in U\left(\sum_{i \in \mathbb{N}} x_{i} \leq y\right)$. Given $y$, we can simultaneously try $\left(q_{0}, q_{1}, \ldots, q_{n}, 0,0, \ldots\right)$ for all rational vectors $\left(q_{0}, \ldots, q_{n}\right)$ such that $y>\sum_{i=0}^{n} q_{i}$. If we find such a vector, then clearly the answer is YES. On the other hand, if any $\left(x_{0}, x_{1}, \ldots\right) \in U$ with $\left(\sum_{i \in \mathbb{N}} x_{i} \leq y\right)$ exists, then there must a rational eventually-zero such vector since $U$ is open.

Given some represented space $\mathbf{X}$, we direct our attention to the space $\mathcal{C}\left(\mathcal{O}(\mathbf{X}), \mathbb{R}_{<}\right)$ of continuous functions from the open subsets of $\mathbf{X}$ to $\mathbb{R}_{<}$. Note that for any $\mu \in \mathcal{C}\left(\mathcal{O}(\mathbf{X}), \mathbb{R}_{<}\right)$we find that if $U \subseteq V$ for some $U, V \in \mathcal{O}(\mathbf{X})$, then $\mu(U) \leq \mu(V)$. We introduce the space $\mathcal{M}(\mathbf{X})$ as a subspace of $\mathcal{C}\left(\mathcal{O}(\mathbf{X}), \mathbb{R}_{<}\right)$by:

$$
\begin{aligned}
\mathcal{M}(\mathbf{X}):=\left\{\mu \in \mathcal{C}\left(\mathcal{O}(\mathbf{X}), \mathbb{R}_{<}\right) \mid \mu(\emptyset)=0 \text { and } \forall\left(U_{i}\right)_{i \in \mathbb{N}} \in \mathcal{C}(\mathbb{N}, \mathcal{O}(\mathbf{X})),\right. \\
\left.\left(\forall i \neq j \in \mathbb{N} U_{i} \cap U_{j}=\emptyset\right) \Rightarrow\left(\mu\left(\bigcup_{i \in \mathbb{N}} U_{i}\right)=\sum_{i \in \mathbb{N}} \mu\left(U_{i}\right)\right)\right\}
\end{aligned}
$$

The space $\mathcal{P}(\mathbf{X})$ of probability measures is obtained in the straightforward way as $\mathcal{P}(\mathbf{X}):=\{\mu \in \mathcal{M}(\mathbf{X}) \mid \mu(\mathbf{X})=1\}$. While it would be more precise to call $\mathcal{M}(\mathbf{X})$ $(\mathcal{P}(\mathbf{X}))$ the space of (probability) valuations, it is well-established that these are the correct notions for computable measure theory. For some discussion and alternative characterizations, see eg Hoyrup and Rojas [25].

Given a point $x \in \mathbf{X}$, we can define the point-measure $\pi_{x}$ by $\pi_{x}(U)=1$ iff $x \in U$ and $\pi_{x}(U)=0$ otherwise. Then $x \mapsto \pi_{x}: \mathbf{X} \rightarrow \mathcal{P}(\mathbf{X})$ is computable. Also, the usual push-forward operation $(f, \mu) \mapsto f^{*} \mu: \mathcal{C}(\mathbf{X}, \mathbf{Y}) \times \mathcal{M}(\mathbf{X}) \rightarrow \mathcal{M}(\mathbf{Y})$ defined via $f^{*} \mu(U)=\mu\left(f^{-1}(U)\right)$ is computable. However, this works only for the continuous functions, not for any larger class of measurable functions: 
Definition 7 Let $\mathcal{P}(\mathbf{X})$ be the space of probability measures on $\mathbf{X}$. To define the space $\mathcal{M}^{C}(\mathbf{X}, \mathbf{Y})$ of measurable functions from $\mathbf{X}$ to $\mathbf{Y}$, identify a measurable function $f: \mathbf{X} \rightarrow \mathbf{Y}$ with its lifted version $f^{*}: \mathcal{P}(\mathbf{X}) \rightarrow \mathcal{P}(\mathbf{Y})$, and define $\mathcal{M}^{C}(\mathbf{X}, \mathbf{Y})$ as the according subspace of $\mathcal{C}(\mathcal{P}(\mathbf{X}), \mathcal{P}(\mathbf{Y}))$.

Proposition $\mathbf{8}$ Let $\mathbf{Y}$ be computably admissible and $\mathbf{X}$ an arbitrary represented space. Then $\mathcal{C}(\mathbf{X}, \mathbf{Y})$ and $\mathcal{M}^{C}(\mathbf{X}, \mathbf{Y})$ are computably isomorphic.

Proof Given a continuous function $f: \mathbf{X} \rightarrow \mathbf{Y}$, we can get $f^{-1}: \mathcal{O}(\mathbf{Y}) \rightarrow \mathcal{O}(\mathbf{X})$, and compose this with a measure $\nu \in \mathcal{P}(\mathbf{X})$ to obtain $f^{*} \nu$. This establishes one direction.

For the other direction, note that $x \mapsto \pi_{x}: \mathbf{X} \rightarrow \mathcal{P}(\mathbf{X})$ is computable, where $\pi_{x}$ is the point measure at $x$. Now $f^{*} \pi_{x}(U)>0 \Leftrightarrow f(x) \in U$. The left hand side is recognizable by the definition of $\mathcal{P}(\mathbf{Y})$, and admissibility of $\mathbf{Y}$ means that the recognizability of the right hand side for arbitrary $U$ implies continuity of $f$, cf Pauly [38, Section 9].

As a representation always is a continuous function (by definition of continuity), we see that we can push a measure on Baire space out to the represented space. As shown by Schröder, in many cases the converse is also true. If we call a represented space $\mathbf{X}$ complete if it admits a total representation $\delta_{\mathbf{X}}: \mathbb{N}^{\mathbb{N}} \rightarrow X$, we can phrase this characterization as follows:

Theorem 9 (Schröder [43]) Let $\mathbf{X}$ be a complete computably admissible space. The map $\mu \mapsto \delta_{\mathbf{X}}^{*} \mu: \mathcal{P}\left(\mathbb{N}^{\mathbb{N}}\right) \rightarrow \mathcal{P}(\mathbf{X})$ is computable and computably invertible.

In the (albeit very restricted) case of probability measures on $\mathbb{N}$ there is a further interesting characterization available. Essentially, one may use typical sequences as names for measures:

Theorem 10 (Pauly [35]) Uniformly in $\varepsilon>0$ there is a computable and computably invertible function $S_{\varepsilon}: \subseteq \mathbb{N}^{\mathbb{N}} \rightarrow \mathcal{P}(\mathbb{N})$ such that for all $\mu \in \mathcal{P}(\mathbb{N})$ we find that $\widehat{\mu}\left(S_{\varepsilon}^{-1}(\mu)\right) \geq 1-\varepsilon$, and if $\nu \neq \mu$, then $\widehat{\nu}\left(S_{\varepsilon}^{-1}(\mu)\right)=0$. Here $\widehat{\mu}$ denotes the induced product measure on $\mathbb{N}^{\mathbb{N}}$.

\subsection{Weihrauch reducibility}

Definition 11 (Weihrauch reducibility) Let $f, g$ be multi-valued functions on represented spaces. Then $f$ is said to be Weihrauch reducible to $g$, in symbols $f \leq_{\mathrm{W}} g$, if there are computable functions $K, H: \subseteq \mathbb{N}^{\mathbb{N}} \rightarrow \mathbb{N}^{\mathbb{N}}$ such that $K\langle$ id, $G H\rangle \vdash f$ for all $G \vdash g$. 
The relation $\leq_{\mathrm{W}}$ is reflexive and transitive. We use $\equiv_{\mathrm{W}}$ to denote equivalence regarding $\leq$ w, and by $<_{\text {w }}$ we denote strict reducibility. By $\mathfrak{W}$ we refer to the partially ordered set of equivalence classes. As shown in Pauly [37] and Brattka and Gherardi [6], W is a distributive lattice, and also the usual product operation on multivalued function induces an operation $\times$ on $\mathfrak{W}$. The algebraic structure on $\mathfrak{W}$ has been investigated in further detail in Higuchi and Pauly [22] and Brattka and Pauly [12].

We will make use of an operation $\star$ defined on $\mathfrak{W}$ that captures aspects of function decomposition. Following Brattka, Gherardi and Marcone [8] and Brattka, Le Roux and Pauly [10], let $f \star g:=\max _{\leq \mathrm{w}}\left\{f_{0} \circ g_{0} \mid f \equiv_{\mathrm{W}} f_{0} \wedge g \equiv_{\mathrm{W}} g_{0}\right\}$. We understand that the quantification is running over all suitable functions $f_{0}, g_{0}$ with matching types for the function decomposition. It is not obvious that this maximum always exists, this is shown in [12] using an explicit construction for $f \star g$. Like function composition, $\star$ is associative but generally not commutative.

An important source for examples of Weihrauch degrees relevant in order to classify theorems are the closed choice principles studied in, eg, Brattka and Gherardi [5] and Brattka, Le Roux and Pauly [4]:

Definition 12 Given a represented space $\mathbf{X}$, the associated closed choice principle $\mathrm{C}_{\mathbf{X}}$ is the partial multivalued function $\mathrm{C}_{\mathbf{X}}: \subseteq \mathcal{A}(\mathbf{X}) \rightrightarrows \mathbf{X}$ mapping a non-empty closed set to an arbitrary point in it.

For any perfect computably compact compact computable metric space $\mathbf{X}$ we find that $\mathrm{C}_{\mathbf{X}} \equiv_{\mathrm{W}} \mathrm{C}_{[0,1]}$ by [4, Corollary 4.5]. For well-behaved spaces, using closed choice iteratively does not increase its power by [4, Theorem 7.3], in particular $\mathrm{C}_{\mathbb{N}} \star \mathrm{C}_{\mathbb{N}} \equiv_{\mathrm{W}} \mathrm{C}_{\mathbb{N}}$ and $\mathrm{C}_{[0,1]} \star \mathrm{C}_{[0,1]} \equiv_{\mathrm{W}} \mathrm{C}_{[0,1]}$. Likewise, it was shown in [4] that $\mathrm{C}_{\mathbb{R}^{n}} \equiv \mathrm{W}_{\mathrm{W}} \mathrm{C}_{\mathbb{R}^{n}} \star \mathrm{C}_{\mathbb{R}^{n}} \equiv_{\mathrm{W}} \mathrm{C}_{\mathbb{N}} \times \mathrm{C}_{[0,1]} \equiv_{\mathrm{W}} \mathrm{C}_{\mathbb{N}} \star \mathrm{C}_{[0,1]} \equiv_{\mathrm{W}} \mathrm{C}_{[0,1]} \star \mathrm{C}_{\mathbb{N}}$ for any $n>0$ Closed choice for $[0,1]$ and $\mathbb{N}$ is incomparable. The degree $C_{[0,1]}$ is closely linked to WKL in reverse mathematics (discussed in [5]), while $C_{\mathbb{N}}$ is Weihrauch-complete for functions computable with finitely many mindchanges ( $\mathrm{cf}[4]$ ).

Further variations of closed principle providing a fruitful area of study are obtained by restriction to certain subclasses of the closed sets. In Brattka, Le Roux and Pauly $[9,10]$ choice for connected closed subsets of $[0,1]^{k}$ was studied (and related to Brouwer's Fixed Point theorem). Convex and finite sets were compared in Le Roux and Pauly [29]. Most related to the present investigation, choice for sets of positive Lebesgue measure was studied in Brattka and Pauly [11] and Brattka, Gherardi and Hölzl [7]. This yields a Weihrauch degree $\mathrm{PC}_{[0,1]}$ with $\mathrm{PC}_{[0,1]}<{ }_{\mathrm{W}} \mathrm{C}_{[0,1]}$ and $\mathrm{PC}_{[0,1]} \mid{ }_{\mathrm{W}} \mathrm{C}_{\mathbb{N}}$. Once more, 
replacing $[0,1]$ with $\{0,1\}^{\mathbb{N}}$ does not change the Weihrauch degree by [11, Corollary 19].

Another typical degree is obtained from the limit operator $\lim : \subseteq \mathbb{N}^{\mathbb{N}} \rightarrow \mathbb{N}^{\mathbb{N}}$ defined via $\lim (p)(n)=\lim _{i \rightarrow \infty} p(\langle n, i\rangle)$. This degree was studied by von Stein [46], Mylatz [32] and Brattka [2, 3], with the latter noting in [3] that it is closely connected to the Borel hierarchy. Hoyrup, Rojas and Weihrauch have shown that lim is equivalent to the RadonNikodym derivative in [26]. It also appears in the context of a model of hypercomputation as shown by Ziegler [49, 48], and captures precisely the additional computational power certain solutions to general relativity could provide beyond computability (Hogarth [23]). It is related to the examples above via $\mathrm{C}_{\mathbb{N}} \times \mathrm{C}_{[0,1]}<_{\mathrm{W}} \lim \equiv_{\mathrm{W}} \lim \times \lim <_{\mathrm{W}} \lim \star \lim$. Important further representatives of the degree of lim are found in the following:

Theorem $13(\mathrm{v}$ Stein [46] $)(\mathrm{id}: \mathcal{A}(\mathbb{N}) \rightarrow \mathcal{V}(\mathbb{N})) \equiv_{\mathrm{W}}(\mathrm{id}: \mathcal{V}(\mathbb{N}) \rightarrow \mathcal{A}(\mathbb{N})) \equiv_{\mathrm{W}}$ $\left(\right.$ id $\left.: \mathbb{R}_{<} \rightarrow \mathbb{R}\right) \equiv_{\mathrm{W}} \lim$

This can be generalized further. For that, we first need to investigate computable embeddability of $\mathbb{N}$ into computable metric spaces. Note that the following result is unusual in the theory of computable metric spaces as it is non-uniform, as opposed to the majority of work, as observed in Rettinger and Weihrauch [40].

Proposition 14 Let $\mathbf{X}$ be an infinite computable metric space. Then there is an embedding of $\mathbb{N}$ into $\mathbf{X}$ computable relative to the Halting problem. If $\mathbf{X}$ has only finitely many isolated points, there even is a computable embedding.

Proof We begin with observing that every isolated point in a computable metric space $\mathbf{X}=\left(X, d,\left(a_{n}\right)_{n \in \mathbb{N}}\right)$ occurs as one of the $a_{n}$. Now we distinguish the two cases:

If there are infinitely many isolated points, then in particular infinitely many of the $a_{n}$ are isolated. Relative to the Halting problem we can decide $\{(n, k) \in \mathbb{N} \mid \exists i \in \mathbb{N} 0<$ $\left.d\left(a_{n}, a_{i}\right)<2^{-k}\right\}$, and thus enumerate a list $\left(a_{n_{j}}, k_{j}\right)$ such that $\forall j, i \in \mathbb{N} \quad d\left(a_{n_{j}}, a_{i}\right)<$ $2^{-k_{j}} \Rightarrow a_{n_{j}}=a_{i}$ and $j \neq j^{\prime} \Rightarrow a_{n_{j}} \neq a_{n_{j^{\prime}}}$. Now $j \mapsto a_{n_{j}}$ is a relatively computable injection from $\mathbb{N}$ to $\mathbf{X}$, and the knowledge of the $k_{j}$ allows to compute the inverse.

If there are only finitely many isolated points, we can assume that their identity (as $a_{i}$ ) is given (as we are not presenting a uniform argument here). As the space is infinite, the remainder has to be a perfect computable metric space. Moreover, if $a_{i} \neq a_{j}$ are both not isolated points, then $B\left(a_{j}, \frac{d\left(a_{i}, a_{j}\right)}{2}\right)$ contains a perfect subset again. We can thus iteratively construct an embedding of $\mathbb{N}$ into $\mathbf{X}$ by searching for two distinct 
non-isolated points, mapping 0 to the first and restricting our future search to a small ball around the second. Then we search for two distinct non-isolated points in that small ball, map 1 to the first and restrict further, and so on.

The following example communicated to the authors by an anonymous referee shows that the relativization in the first case of Proposition 14 is not dispensable:

Example 15 There is an infinite computable Polish space $\mathbf{X}$ such that $\mathbb{N}$ does not computably embed into $\mathbf{X}$.

Proof Let $x \in\{0,1\}^{\mathbb{N}}$ be a 1-generic computable relative to the Halting problem. By the Shoenfield limit lemma there is a computable sequence $\left(x_{n}\right)_{n \in \mathbb{N}}$ converging to $x$. Let $X=\{x\} \cup\left\{x_{n} \mid x \in \mathbb{N}\right\}$, and $d$ be the restriction of the usual metric on Cantor space. Then $\mathbf{X}=\left(X, d,\left(x_{n}\right)_{n \in \mathbb{N}}\right)$ is a complete computable metric space.

Let $\iota: \mathbb{N} \rightarrow \mathbf{X}$ be a computable injection. As $x$ is not a computable point, $x$ cannot be in the range of $\iota$ (denoted by $\iota[\mathbb{N}]$ ). Now assume that $\iota$ had a computable inverse. Then for any $\iota(i)=x_{n}$ there must be some $k_{x_{n}} \in \mathbb{N}$ computable from $x_{n}$ such that $B\left(x_{n}, 2^{-k_{x_{n}}}\right) \cap \iota[\mathbb{N}]=\left\{x_{n}\right\}$. But then we can compute the open set $U=\bigcup_{i \in \mathbb{N}} B\left(\iota(i), 2^{-k_{\iota(i)}}\right)$. As $x$ is the limit point of the $x_{n}$, in particular we find that $x$ has to lie on the boundary of $U$, but this contradicts $x$ being a 1 -generic. Thus no computable injection $\iota: \mathbb{N} \rightarrow \mathbf{X}$ can have a computable inverse.

Proposition 16 Let there be a computable embedding of $\mathbb{N}$ into a computable metric space $\mathbf{X}$. Then:

$$
\lim \leq_{\mathrm{W}}(\mathrm{id}: \mathcal{A}(\mathbf{X}) \rightarrow \mathcal{V}(\mathbf{X}))
$$

Proof There being a computable embedding of $\mathbb{N}$ into $\mathbf{X}$ translates into the existence of a computable sequence $a \in \mathcal{C}(\mathbb{N}, \mathbf{X})$ together with a computable sequence $r \in \mathbb{N}^{\mathbb{N}}$ such that $\forall n, m \in \mathbb{N}\left(n \neq m \Rightarrow d\left(a_{n}, a_{m}\right)>2^{-r_{n}}\right)$.

We show the reduction $($ id $: \mathcal{A}(\mathbb{N}) \rightarrow \mathcal{V}(\mathbb{N})) \leq_{\mathrm{W}}(\mathrm{id}: \mathcal{A}(\mathbf{X}) \rightarrow \mathcal{V}(\mathbf{X}))$ instead. Given some closed set $A \in \mathcal{A}(\mathbb{N})$, we construct a closed set $B \in \mathcal{A}(\mathbf{X})$ as follows: When $n \in \mathbb{N}$ is removed from $A$, we remove $B\left(a_{n}, 2^{-r_{n}}\right)$ from $B$. By choice of the sequence, we then find that $n \in A \Leftrightarrow B\left(x_{n}, 2^{-r_{n}}\right) \cap B \neq \emptyset$, hence knowing $B$ as an overt set implies knowing $A$ as an overt set.

Corollary 17 Let $\mathbf{X}$ be an infinite computable metric space. Then

$$
\lim \leq_{\mathrm{W}}(\mathrm{id}: \mathcal{A}(\mathbf{X}) \rightarrow \mathcal{V}(\mathbf{X}))
$$

relative to the Halting problem. 
Recall that a computable metric space $\mathbf{X}$ is called effectively locally compact if we have a standard basis $\left(B_{n}\right)_{n \in \mathbb{N}}$ available such that the closures $\bar{B}_{n}$ form a computable sequence in $\mathcal{K}(\mathbf{X})$. Note that any locally compact computable metric space is effectively locally compact relative to some oracle.

Proposition 18 Let $\mathbf{X}$ be an effectively locally compact computable metric space. Then:

$$
(\text { id }: \mathcal{A}(\mathbf{X}) \rightarrow \mathcal{V}(\mathbf{X})) \leq_{\mathrm{W}} \lim
$$

Proof Given $A \in \mathcal{A}(\mathbf{X})$, we can enumerate all basic open balls $B_{n}$ such that $\overline{B_{n}} \cap A=\emptyset$. Using (id : $\mathcal{A}(\mathbb{N}) \rightarrow \mathcal{V}(\mathbb{N})$ ) $\equiv_{\mathrm{W}}$ lim, we can transform such an enumeration to its characteristic function $\chi \in\{0,1\}^{\mathbb{N}}$ (ie $\chi_{n}=1 \Leftrightarrow \overline{B_{n}} \cap A=\emptyset$ ).

Now if for some open $U \in \mathcal{O}(\mathbf{X})$ we have $U \cap A \neq \emptyset$, then there is some $B_{n}$ with $\overline{B_{n}} \subseteq U$ and $\overline{B_{n}} \cap A \neq \emptyset$. Given $\chi$, we can effectively search for such a candidate, thus, $A \in \mathcal{V}(\mathbf{X})$ is computable from $\chi$.

Corollary 19 Let $\mathbf{X}$ be an infinite locally compact computable metric space. Then relative to some oracle:

$$
(\mathrm{id}: \mathcal{A}(\mathbf{X}) \rightarrow \mathcal{V}(\mathbf{X})) \equiv_{\mathrm{W}} \lim
$$

Proposition 20 Let $\mathbf{X}$ admit a computable sequence $\left(a_{n}\right)_{n \in \mathbb{N}}$ such that:

$$
\forall n \in \mathbb{N} \quad a_{n} \notin \overline{\left\{a_{i} \mid i \neq n\right\}}
$$

Then:

$$
\lim \leq_{\mathrm{W}}(\mathrm{id}: \mathcal{V}(\mathbf{X}) \rightarrow \mathcal{A}(\mathbf{X}))
$$

Proof As the (closure of the) image of an overt set under a continuous function is overt, the map $A \mapsto \overline{\left\{a_{i} \mid i \in A\right\}}: \mathcal{V}(\mathbb{N}) \rightarrow \mathcal{V}(\mathbf{X})$ is computable. By assumption on $\left(a_{n}\right)_{n \in \mathbb{N}}$, we now find that $n \notin A \Leftrightarrow a_{n} \notin \overline{\left\{a_{i} \mid i \in A\right\}}$. Thus, we have a reduction:

$$
(\text { id }: \mathcal{V}(\mathbb{N}) \rightarrow \mathcal{A}(\mathbb{N})) \leq_{\mathrm{W}}(\mathrm{id}: \mathcal{V}(\mathbf{X}) \rightarrow \mathcal{A}(\mathbf{X}))
$$

By v Stein's result (Theorem 13), this is equivalent to our claim.

Proposition 21 Let $\mathbf{X}$ be a computable metric space. Then:

$$
(\text { id }: \mathcal{V}(\mathbf{X}) \rightarrow \mathcal{A}(\mathbf{X})) \leq{ }_{\mathrm{w}} \lim
$$

Proof We use the characterization of $\mathcal{O}(\mathbf{X})$ via some basis $U_{n}$ as provided in Proposition 3. Given $A \in \mathcal{V}(\mathbf{X})$, we may compute $L:=\left\{n \in \mathbb{N} \mid U_{n} \cap A \neq \emptyset\right\} \in \mathcal{O}(\mathbb{N}) \equiv \mathcal{V}(\mathbb{N})$. Using (id $: \mathcal{V}(\mathbb{N}) \rightarrow \mathcal{A}(\mathbb{N})$ ), we find $L \in \mathcal{A}(\mathbb{N})$, or equivalently, $L^{C} \in \mathcal{O}(\mathbb{N})$. Now we may compute $\left(\bigcup_{i \in L^{C}} U_{i}\right) \in \mathcal{O}(\mathbf{X})$, and will find this to be equivalent to $A \in \mathcal{A}(\mathbf{X})$. 
Corollary 22 If $\mathbf{X}$ is an infinite computable metric space, then

$$
\lim \equiv_{\mathrm{W}}(\mathrm{id}: \mathcal{V}(\mathbf{X}) \rightarrow \mathcal{A}(\mathbf{X}))
$$

relative to the Halting problem. If $\mathbf{X}$ has only finitely many isolated points, the claim holds true without an oracle.

Proof One direction holds always computably by Proposition 21. For the other direction, note that Proposition 14 ensures that we can invoke the (relativization of) Proposition 20.

Corollary $23($ id $: \mathcal{A}([0,1]) \rightarrow \mathcal{V}([0,1])) \equiv_{\mathrm{W}}(\mathrm{id}: \mathcal{V}([0,1]) \rightarrow \mathcal{A}([0,1])) \equiv_{\mathrm{W}} \lim$

\subsection{Hausdorff dimension and the Frostman lemma}

The Frostman lemma essentially states that having positive Hausdorff dimension is equivalent to admitting a measure that is far from being atomic - and being far from atomic is given a quantitative interpretation and exactly tied to the Hausdorff dimension. We introduce the Hausdorff dimension only as a property of closed subsets of $[0,1]$ here:

Definition 24 Given some $A \in \mathcal{A}([0,1])$, we define its Hausdorff $\operatorname{dimension}_{\operatorname{dim}_{\mathcal{H}}(A)}$ as:

$$
\begin{aligned}
\operatorname{dim}_{\mathcal{H}}(A) & :=\inf \left\{d \geq 0 \mid \inf \left\{\sum_{i \in \mathbb{N}} r_{i}^{d} \mid \exists\left(x_{i}\right)_{i \in \mathbb{N}} A \subseteq \bigcup_{i \in \mathbb{N}} B\left(x_{i}, r_{i}\right)\right\}=0\right\} \\
& =\sup \left\{d \geq 0 \mid \lim _{\delta \rightarrow 0} C_{A, d, \delta}=\infty\right\}
\end{aligned}
$$

where:

$$
C_{A, d, \delta}:=\inf \left\{\sum_{i \in \mathbb{N}} r_{i}^{d} \mid \exists\left(x_{i}\right)_{i \in \mathbb{N}} \quad A \subseteq \bigcup_{i \in \mathbb{N}} B\left(x_{i}, r_{i}\right) \wedge \forall i r_{i}<\delta\right\}
$$

We find $\operatorname{dim}_{\mathcal{H}}(A) \in[0,1]$ for all $A \in \mathcal{A}([0,1]), \operatorname{dim}_{\mathcal{H}}(A)=0$ for any countable $A$ and $\operatorname{dim}_{\mathcal{H}}(A)=1$ whenever $\lambda(A)>0$ where $\lambda$ denotes the Lebesgue measure.

Proposition 25 Given a sequence $\left(d_{i}\right)_{i \in \mathbb{N}}$ of reals with $d_{i}>2$, we can compute some $C \in \mathcal{A}([0,1])$ with $\operatorname{dim}_{\mathcal{H}}(C)=\liminf _{n \in \mathbb{N}} \frac{\ln 2}{\ln d_{n}}$.

Proof This is adapted from a construction provided in Fouché, Mukeru and Davie [17, Page 11]: we define a family $\left(\left[a_{w}, b_{w}\right]\right)_{w \in\{0,1\}^{*}}$ of intervals indexed by $\{0,1\}^{*}$ by $\left[a_{\varepsilon}, b_{\varepsilon}\right]=[0,1], a_{w 0}=a_{w}, b_{w 0}=a_{w}+d_{|w|}^{-1}\left(b_{w}-a_{w}\right), a_{w 1}=a_{w}+\left(1-d_{|w|}^{-1}\right)\left(b_{w}-a_{w}\right)$, $b_{w 1}=b_{w}$. Then define $C:=\bigcap_{n \in \mathbb{N}} \bigcup_{w \in\{0,1\}^{n}}\left[a_{w}, b_{w}\right]$. It is a standard (but not trivial) calculation to verify that $\operatorname{dim}_{\mathcal{H}}(C)=\liminf _{n \in \mathbb{N}} \frac{\ln 2}{\ln d_{n}}$, see eg Falconer [16]. 
Corollary 26 Given $x \in[0,1]$ we can compute some $A \in \mathcal{A}([0,1])$ with $\operatorname{dim}_{\mathcal{H}}(A)=$ $x$.

Proof From $x \in[0,1]$ we can compute some sequence $\left(d_{i}\right)_{i \in \mathbb{N}}$ of reals with $d_{i}>2$ and $\lim \inf _{n \in \mathbb{N}} \frac{\ln 2}{\ln d_{n}}=x$. Now invoke Proposition 25 .

While having positive Hausdorff dimension (and in particular having Hausdorff dimension 1) is an indicator of a set being large, it is a rather weak indicator as the following shows:

Theorem 27 (Shmerkin $\left.[44]^{3}\right)$ There is a computable surjection $\vartheta:[0,1] \rightarrow[0,1]$ such that $\forall x \in[0,1] \operatorname{dim}_{\mathcal{H}}\left(\vartheta^{-1}(\{x\})\right)=1$.

We can now introduce Frostman measures, and state the Frostman lemma:

Definition 28 An $s$-Frostman measure (on $[0,1]$ ) is a non-zero Radon measure $\mu$ such that for any $x, r \in[0,1]$ we find $\mu(B(x, r)) \leq r^{s}$.

Lemma 29 (Frostman [18])

$$
\operatorname{dim}_{\mathcal{H}}(A)=\sup _{s \in[0,1]}\{\exists \mu \mid \operatorname{supp}(\mu) \subseteq A \wedge \mu \text { is an } s \text {-Frostman measure }\}
$$

We will in particular investigate the Weihrauch degree of the following maps:

Definition 30 Let Frost $: \subseteq \mathcal{A}([0,1]) \times[0,1] \rightrightarrows \mathcal{M}([0,1])$ be defined via $\mu \in$ Frost $(A, s)$ iff $\mu$ is an $s$-Frostman measure with $\operatorname{supp}(\mu) \subseteq A$. Let StrictFrost $: \subseteq$ $\mathcal{A}([0,1]) \times[0,1] \rightrightarrows \mathcal{M}([0,1])$ be defined via $\mu \in \operatorname{StrictFrost}(A, s)$ iff $\mu$ is an $s$ Frostman measure with $\operatorname{supp}(\mu)=A$.

\section{Measures and support}

We shall begin by investigating how a measure and its support are related. We show that the support is fundamentally an overt set, rather than a closed set; and that both obtaining the support of a given measure as a closed set, and constructing a measure with support as a given closed set are equivalent to the lim-operator. The measures constructed here will generally fail to be non-atomic.

\footnotetext{
${ }^{3}$ While the statement there is only about a continuous surjection, it is obvious that the constructed map is even computable.
} 
Theorem 31 supp : $\mathcal{M}(\mathbf{X}) \rightarrow \mathcal{V}(\mathbf{X})$ is computable. If $\mathbf{X}$ is a computable Polish space, it has a computable multivalued inverse.

Proof Note that an open set $U$ intersects $\operatorname{supp}(\mu)$ iff $\mu(U)>0$. It is easy to see that $\chi_{>0}: \mathbb{R}_{<} \rightarrow \mathbb{S}$ is computable. Taking into consideration the definitions of $\mathcal{M}$ and $\mathcal{V}$, we see that every realizer of $\mu \mapsto\left(U \mapsto \chi_{>0}(\mu(U))\right)$ is a realizer of supp; and since the former has a computable realizer, so has the latter.

For the reverse direction ${ }^{4}$ we use Proposition 4 to express the given overt set as the closure of a sequence $\left(x_{i}\right)_{i \in \mathbb{N}}$. Now we can compute a sum of point measures via $\mu(U)=\sum_{\left\{i \in \mathbb{N} \mid x_{i} \in U\right\}} 2^{-i}$. Clearly $\operatorname{supp}(\mu)=\overline{\left\{x_{i} \mid i \in \mathbb{N}\right\}}$. While the given overt set could be empty this is unproblematic: Simply start outputting the zero measure, until the input set has been observed to be non-empty, then switch to the measure as constructed above.

Corollary 32 Let $\mathbf{X}$ be a computable Polish space. Given a non-zero measure $\mu \in \mathcal{M}(\mathbf{X})$, we can compute a point $x \in \operatorname{supp}(\mu)$.

Proof Combine Corollary 5 with Theorem 31.

Corollary 33 Consider $\operatorname{supp}_{\mathbf{X}}^{\mathcal{A}}: \mathcal{M}(\mathbf{X}) \rightarrow \mathcal{A}(\mathbf{X})$ and $\left(\operatorname{supp}_{\mathbf{X}}^{\mathcal{A}}\right)^{-1}: \mathcal{A}(\mathbf{X}) \rightrightarrows \mathcal{M}(\mathbf{X})$ for an infinite effectively locally compact computable Polish space $\mathbf{X}$. Then

$$
\operatorname{supp}_{\mathbf{X}}^{\mathcal{A}} \equiv_{\mathrm{W}}\left(\operatorname{supp}_{\mathbf{X}}^{\mathcal{A}}\right)^{-1} \equiv_{\mathrm{W}} \lim
$$

relative to the Halting problem. If $\mathbf{X}$ admits a computable embedding of $\mathbb{N}$, in particular, if $\mathbf{X}$ is perfect, the claim is true without an oracle.

Proof Using Theorem 31 we find

$$
\operatorname{supp}_{\mathbf{X}}^{\mathcal{A}} \equiv_{\mathrm{W}}(\mathrm{id}: \mathcal{V}(\mathbf{X}) \rightarrow \mathcal{A}(\mathbf{X})) \wedge\left(\operatorname{supp}_{\mathbf{X}}^{\mathcal{A}}\right)^{-1} \equiv_{\mathrm{W}}(\mathrm{id}: \mathcal{A}(\mathbf{X}) \rightarrow \mathcal{V}(\mathbf{X}))
$$

By Corollary 22 the former map is equivalent to lim relative to the Halting problem. As $\mathbf{X}$ is effectively locally compact, the Halting problem suffices as oracle in Corollary 19 , which then gives the equivalence to $\lim$ of (id $: \mathcal{A}(\mathbf{X}) \rightarrow \mathcal{V}(\mathbf{X})$ ). As the Halting problem is only used to ensure existence of a relatively computable embedding of $\mathbb{N}$ through Proposition 14, with the extra assumption, everything holds computably.

\footnotetext{
${ }^{4}$ This part of the proof was greatly simplified based on suggestions by an anonymous referee.
} 
If we just demand that the (non-zero) measure to be constructed is supported by the given (non-empty) closed set (ie $\operatorname{supp}(\mu) \subseteq A$ rather than $\operatorname{supp}(\mu)=A$ ), the resulting operator ConstructMeasure $\mathbf{X}: \subseteq \mathcal{A}(\mathbf{X}) \rightrightarrows \mathcal{M}(\mathbf{X})$ is strictly simpler for many spaces:

Theorem 34 Let $\mathbf{X}$ be a computable Polish space. Then ConstructMeasure ${ }_{\mathbf{X}} \equiv_{\mathrm{W}} \mathrm{C}_{\mathbf{X}}$.

Proof For ConstructMeasure ${ }_{\mathbf{X}} \leq_{\mathrm{W}} \mathrm{C}_{\mathbf{X}}$, use $\mathrm{C}_{\mathbf{X}}$ to pick a point $x$ in $A$, and then compute the point measure $\mu_{x}$, which is non-zero and satisfies $\operatorname{supp}\left(\mu_{x}\right)=\{x\} \subseteq A$.

For the other direction, we need to show that given a non-zero measure $\mu$ supported by $A$ we can compute some point $x \in A$. We do this by searching for a basic open ball $B\left(x_{1}, 2^{-1}\right)$ with $\mu\left(B\left(x_{1}, 2^{-1}\right)\right)>0$, which we will find eventually. Then we search for some $x_{2} \in B\left(x_{1}, 2^{-1}\right)$ such that $\mu\left(B\left(x_{2}, 2^{-2}\right)\right)>0$, which also will eventually be detected. We continue to produce a fast Cauchy sequence, of which we can compute the limit $x$. Now $x \in A$ is easy to see.

Corollary 35 ConstructMeasure $[0,1]<\mathrm{W}\left(\operatorname{supp}_{[0,1]}^{\mathcal{A}}\right)^{-1}$

Proof We use Corollary 33, Theorem 34 and $\mathrm{C}_{[0,1]}<_{\mathrm{W}} \lim ($ eg from Brattka and Gherardi [6, Theorem 7.13]).

\section{Non-atomic measures}

The picture painted in Section 3 of the constructivity (or lack thereof) of constructing measures crucially depends on the option of resulting measures having atoms, ie single points carrying positive measure. In the present section we first introduce the notion of flows on infinite trees as a technical tool (which could be of some interest in its own right). We then proceed to investigate the role of overtness, which drastically differs from the results above. Considering some Weihrauch degrees related to Hausdorff dimension then leads up to the Frostman lemma.

\subsection{Non-atomic measures on $[0,1]$ and flows}

We consider assignments of non-negative real numbers to the edges of a full infinite binary tree, ie the space $\left(\mathbb{R}_{0}^{+}\right)^{\{0,1\}^{*}}$. Here $\mathbb{R}_{0}^{+}$is just the subspace of $\mathbb{R}$ containing the non-negative numbers. Such an assignment $f$ is called a flow if $\forall v \in\{0,1\}^{*} f(v)=$ $f(v 0)+f(v 1)$. We extend $\leq$ to $\left(\mathbb{R}_{0}^{+}\right)^{\{0,1\}^{*}}$ in a point-wise fashion, and let $\varepsilon \in\{0,1\}^{*}$ denote the empty word. 
Definition 36 The multivalued map MaxFlow : $\left(\mathbb{R}_{0}^{+}\right)^{\{0,1\}^{*}} \rightrightarrows\left(\mathbb{R}_{0}^{+}\right)^{\{0,1\}^{*}}$ is defined via $g \in \operatorname{MaxFlow}(f)$ iff $g \leq f, g$ is a flow and $g(\varepsilon)$ is maximal under these conditions.

Theorem 37 MaxFlow $\equiv_{\mathrm{W}} \lim$

Proof To show lim $\leq_{\mathrm{W}}$ MaxFlow, we prove $\left.\operatorname{id}_{\mathbb{R}_{>}, \mathbb{R}}\right|_{\left\{x \in \mathbb{R}_{>} \mid x \geq 0\right\}} \leq_{\mathrm{W}}$ MaxFlow instead. Let the input to $\left.\operatorname{id}_{\mathbb{R}_{>}, \mathbb{R}}\right|_{\left\{x \in \mathbb{R}_{>} \mid x \geq 0\right\}}$ be the decreasing sequence $\left(q_{i}\right)_{i \in \mathbb{N}}$ of non-negative rationals. Now we construct an assignment via $f\left(1^{n}\right)=q_{n}$ and $f(v)=0$ for $v \neq 1^{|v|}$. Now if $g \in \operatorname{MaxFlow}(f)$, then $g(\varepsilon)=\inf _{i \in \mathbb{N}} q_{i}=\lim _{i \rightarrow \infty} q_{i}$.

For the other direction, we inductively define a decreasing sequence of real numbers for each edge of the tree by setting $a_{0}^{v}=f(v)$ and $a_{n+1}^{v}=\min \left\{a_{n}^{v}, a_{n}^{v 0}+a_{n}^{v 1}\right\}$. Then $\left(v \mapsto \lim _{i \rightarrow \infty} a_{i}^{v}\right) \in \operatorname{MaxFlow}(f)$, so using lim countably many times in parallel suffices to find a valid max flow. As stated above, this is equivalent to using lim just once.

Definition 38 The multivalued map NonZeroFlow $: \subseteq\left(\mathbb{R}_{0}^{+}\right)^{\{0,1\}^{*}} \rightrightarrows\left(\mathbb{R}_{0}^{+}\right)^{\{0,1\}^{*}}$ is defined via $g \in$ NonZeroFlow $(f)$ iff $g \leq f, g$ is a flow and $g(\varepsilon)>0$.

Theorem 39 NonZeroFlow $\equiv_{\mathrm{W}} \mathrm{C}_{\mathbb{N}} \times \mathrm{C}_{\{0,1\}^{\mathbb{N}}}$.

Proof To see that NonZeroFlow $\leq_{\mathrm{W}} \mathrm{C}_{\mathbb{N}} \times \mathrm{C}_{\{0,1\}^{\mathbb{N}}}$ we use a non-deterministic algorithm following [4]. We guess some number $k \in \mathbb{N}$ together with an assignment $g:\{0,1\}^{*} \rightarrow[0,1]$ where $g(\varepsilon)=2^{-k}$ (note that $g$ is an element of a computably compact computable metric space). If $g$ is not a flow with $g \leq f$ where $f$ is the input to NonZeroFlow, we will detect this eventually.

For the other direction, we may prove WKL $\times$ UpperBound $\leq \mathrm{w}$ NonZeroFlow instead. Here WKL is the problem to find an infinite path through a given infinite binary tree $T$, and is equivalent to $C_{\{0,1\}^{\mathbb{N}}}$ for trivial reasons, cf Gherardi and Marcone [19]. The problem UpperBound is given a monotone and bounded sequence of natural numbers $\left(n_{i}\right)_{i \in \mathbb{N}}$, the goal is to find some upper bound; see eg Pauly, Davie and Fouché [39, Lemma 3]. Let $\lambda_{n}^{T}:=|\{v \in T|| v \mid=n\}|-1$. We define an assignment $f:\{0,1\}^{*} \rightarrow[0,1]$ via $f(v)=2^{-n_{|v|}-\lambda_{|v|}^{T}}$ if $v \in T$ and $f(v)=0$ otherwise. Any non-zero flow $g$ smaller than $f$ then computes both an infinite path through $T$ (just go down some path carrying positive flow) and an upper bound for $\left(n_{i}\right)_{i \in \mathbb{N}}$ (in form of $N$ s.t. $g(\varepsilon)>2^{-N}$ ).

Say that a flow $f$ is concentrated iff for all $v \in\{0,1\}^{*}$ either $f(v) \geq 2^{-2|v|-1}$ or $f(v)=0$. Let the multivalued map ConcentrateFlow map a non-zero flow $f$ to any non-zero concentrated flow $g$ with $f(\varepsilon) g \leq f$. 
Proposition 40 ConcentrateFlow is computable.

Proof We may normalize the input flow to $f(\varepsilon)=1$. We define $g$ iteratively, starting with $g(\varepsilon)=\frac{1}{2}$. We want to ensure the invariant $g(v)>0 \Rightarrow\left(g(v)+2^{-2|v|-1} \leq f(v)\right) \wedge$ $\left(2^{-2|v|-1} \leq g(v)\right)$ throughout the process; any flow constructed this way clearly is a valid answer. The invariant holds at the initial step.

For the continuation step, we can computably select a true case among the following:

$\left(f(v 0) \leq 3 \cdot 2^{-2|v|-3}\right) \quad$ We set $g(v 0)=0$ and $g(v 1)=g(v)$. The invariant is trivially satisfied for $v 0$. For $v 1$, we need to check that $g(v 1)+2^{-2|v 1|-1} \leq f(v 1)$. The case we are in gives us $f(v 1) \geq f(v)-3 \cdot 2^{-2|v|-3}$, thus it suffices to argue that $g(v)+2^{-2|v|-3} \leq f(v)-3 \cdot 2^{-2|v|-3}$ given $g(v)+2^{-2|v|-1} \leq f(v)$. This just follows from $2^{-2|v|-3}+3 \cdot 2^{-2|v|-3}=2^{-2|v|-1}$.

$\left(f(v 1) \leq 3 \cdot 2^{-2|v|-3}\right) \quad$ We set $g(v 0)=g(v)$ and $g(v 1)=0$. The invariant is preserved just as in the case $f(v 0) \leq 3 \cdot 2^{-2|v|-3}$.

$\left(f(v 0) \geq 2^{-2|v|-2} \wedge f(v 1) \geq 2^{-2|v|-2}\right) \quad$ Here we set

$$
(v 0)=\min \left\{f(v 0)-2^{-2|v|-3}, g(v)-2^{-2|v|-3}\right\}
$$

and $g(v 1)=g(v)-g(v 0)$. The first part of the invariant is satisfied for $g(v 0)$ by construction. We find $g(v 1)+2^{-2|v 1|-1}=g(v)-g(v 0)+2^{-2|v|-3}$, and need to show that this expression is bounded by $f(v 1)$ from above. If $g(v 0)=g(v)-2^{-2|v|-3}$, then this follows from $2^{-2|v|-2} \leq f(v 1)$ by the assumption of the case. If $g(v 0)=f(v 0)-2^{-2|v|-3}$, then the desired inequality is equivalent to $g(v)+2^{-2|v|-2} \leq f(v 0)+f(v 1)=f(v)$, and thus follows from the invariant for $v$.

By assumption $2^{-2|v|-1} \leq g(v)$, thus $2^{-2|v 0|-1} \leq g(v)-3 \cdot 2^{-2|v|-3} \leq g(v 0)$. Also, $g(v 1)=g(v)-g(v 0) \geq 2^{-2|v|-3}$, thus the second part of the invariant is satisfied for both values.

Call a flow $f$ non-atomic if $\lim _{n \rightarrow \infty} f\left(p_{\leq n}\right)=0$ for any $p \in\{0,1\}^{\mathbb{N}}$. The reason for our interest in flows is a connection between non-atomic flows and non-atomic measures on $[0,1]$. Given $v \in\{0,1\}^{*}$, let $\mathfrak{D}_{v}$ be the associated closed dyadic interval. If $\mu$ is a non-atomic measure on $[0,1]$ with known value $\mu([0,1])$, then $f_{\mu}(v):=\mu\left(\mathfrak{D}_{v}^{\circ}\right)$ defines a non-atomic flow. Conversely, any non-atomic flow $f$ induces a non-atomic measure $\mu_{f}$ as follows: Given some open set $U$, we can enumerate all $v$ with $\mathfrak{D}_{v} \subseteq U$. By skipping entries as required, we can turn this enumeration into a prefix-free list $\left(v_{i}^{U}\right)_{i \in \mathbb{N}}$. We find that $U \backslash\left(\bigcup_{i \in \mathbb{N}} \mathfrak{D}_{v_{i}^{U}}\right)$ contains only dyadic rationals. We can define $\mu_{f}(U)=\sum_{i \in \mathbb{N}} f\left(v_{i}^{U}\right)$. 


\subsection{Overtness and non-atomic measures}

It is clear that isolated points cannot be part of the support of a non-atomic measure. Thus, when constructing measures supported by given sets, we either need to consider only perfect sets, or be satisfied if the support is included in the set, rather than demanding equality. In the latter situation, overtness becomes useless as demonstrated next:

Given some closed set $A$, let $A^{*}$ be the largest perfect set contained in $A$. Alternatively, let $A^{*}$ be the set resulting from $A$ after removing the isolated points $\alpha$ times for some countable ordinal $\alpha$, after which no isolated points remain.

Definition 41 Define PerfectCore $: \mathcal{A}(\mathbb{R}) \rightrightarrows \mathcal{A}(\mathbb{R}) \wedge \mathcal{V}(\mathbb{R})$ by $B \in \operatorname{PerfectCore}(A)$ if $B \supseteq A$ and $B^{*}=A^{*}$.

Proposition 42 PerfectCore is computable.

Proof We shall consider only dyadic intervals as basic open intervals, ie intervals of the form $I_{m, n, k}:=\left(\frac{n}{2^{m}}, \frac{n+k}{2^{m}}\right)$ for $n \in \mathbb{Z}, k, m \in \mathbb{N}, k<2^{m}$. Note that the restriction on $k$ implies that there are locally only finitely many large intervals (for any fixed notion of large). We can assume that the input set $A$ is given via an enumeration $\left(n_{i}\right)_{i \in \mathbb{N}}$ denoting its complement via $A^{C}=\bigcup_{i \in \mathbb{N}} I_{n_{i}}$. Let $U_{t}:=\bigcup_{i=0}^{t} I_{n_{i}}$. Note that relative to $\left(n_{i}\right)_{i \in \mathbb{N}}$, it is decidable whether or not $I_{s} \subseteq U_{t}$ for $s, t \in \mathbb{N}$.

Our goal is to decide for each basic open interval whether it intersects the constructed output $B$. The enumeration of all basic open intervals intersecting $B$ constitutes a name for $B \in \mathcal{V}(\mathbb{R})$, and the enumeration of all basic open intervals not intersecting $B$ constitutes a name for $B \in \mathcal{A}(\mathbb{R})$.

We maintain as auxiliary structures a set $X$ of rational numbers and a set $M$ of basic open intervals. We only add points to $X$, but never remove them, while intervals may be added to or removed from $M$. At any stage of the construction, both $X$ and $M$ will be finite (this ensures that testing conditions for all elements of $X$ or $M$ respectively is unproblematic), and both are initially empty. We let $B_{t}^{C}$ be the union of all those $I_{s}$ for which have decided that $I_{s} \cap B=\emptyset$ at a step prior to $t$.

In order to make our decision on $I_{t}$, we go through the following steps, and use the first applicable one:

(1) If $I_{t} \subseteq B_{t}^{C}$, then $I_{t} \cap B=\emptyset$.

(2) If $X \cap I_{t} \neq \emptyset$, then $I_{t} \cap B \neq \emptyset$. 
(3) If $I_{t} \subseteq U_{t}$ and there is some $I_{s} \in M$ with $I_{t} \cap I_{s} \cap B_{t} \neq \emptyset$, then we pick some dyadic rational $x \in I_{t} \cap I_{s} \cap B_{t}$. We add $x$ to $X$, remove $I_{s}$ from $M$ and we decide that $I_{t} \cap B \neq \emptyset$. Let $I_{t_{1}}$ and $I_{t_{2}}$ be the two connectedness components of $I_{t} \backslash\{x\}$. We decide that $I_{t_{1}} \cap B=\emptyset$ and $I_{t_{2}} \cap B=\emptyset$. (If there are multiple applicable $I_{s}$ in $M$, we pick points in each of them, decide that the finitely dyadic intervals the removal of these points splits $I_{t}$ into do not intersect $B$, and remove all applicable $I_{s}$ from $M$.)

(4) If $I_{t} \subseteq U_{t}$, then we decide that $I_{t} \cap B=\emptyset$.

(5) Otherwise, we add $I_{t}$ to $M$ and decide that $I_{t} \cap B \neq \emptyset$.

In order to show that this algorithm correctly solves PerfectCore, we will argue that $A \cup X$ (where $X$ here refers to the final state, ie the set of all points ever added to $X$ ) is a closed set, that the algorithm produces a $\mathcal{A}(\mathbb{R}) \wedge \mathcal{V}(\mathbb{R})$-name of $B=A \cup X$, and that all points of $X$ are isolated in $A \cup X$, hence $A \cup X \in \operatorname{PerfectCore}(A)$.

Claim: All points in $X$ are isolated in $A \cup X$.

A point $x \in X$ must have been added to $X$ in Step (3), and is contained in some $I_{t} \subseteq U_{t}$. As $\bigcup_{t \in \mathbb{N}} U_{t}=A^{C}$, we know that $I_{t} \cap A=\emptyset$, thus $x$ cannot be an accumulation point of $A$. Moreover, for Step (3) to have been reached, the interval $I_{t}$ cannot contain any points from $X$ added prior to $x$. As we decide that $I_{t_{1}} \cap B=I_{t_{2}} \cap B=\emptyset$, we find that $I_{t_{1}} \cup I_{t_{2}} \subseteq B_{s}^{C}$ for any $s>t$. If some point $y$ gets added to $X$ at stage $s>t$, this prevents that $y \in I_{t_{1}} \cup I_{t_{2}}$, hence $x$ is even isolated in $A \cup X$.

Claim: $A \cup X$ is a closed set.

As $A$ is closed and $X$ contains only isolated points, we will argue that all accumulation points of $X$ are in $A$. For this, it suffices to show that if $I_{s}$ is some basic interval with $\bar{I}_{s} \cap A=\emptyset$, then $I_{s} \cap X$ is finite. Since $\bar{I}_{s} \cap A=\emptyset$, by compactness there is some sufficiently large $t$ such that $\bar{I}_{s} \subseteq U_{t}$. At time $t$, only finitely many points have been added to $X$, and finitely many intervals are in $M$ (and subsequently can cause addition of points to $X$ ). Further points can be added to $X$ only if an interval containing them are added to $M$. But there are only finitely many basic dyadic intervals that intersect $I_{s}$ yet are not contained in $U_{t}$. Those that are contained in $U_{t}$ cannot be added to $M$ after step $t$.

Claim: If $I_{t} \cap(A \cup X)=\emptyset$, we decide that $I_{t}$ does not intersect $B$.

Any decision that $I_{t} \cap B \neq \emptyset$ is justified by Step (2), (3) or (5). If Step (2) or (3) are applicable for $I_{t}$, then afterwards we will have $I_{t} \cap X \neq \emptyset$. If $I_{t} \cap B \neq \emptyset$ is decided from Step (5), then $I_{t}$ is added to $M$. If $I_{t}$ ever gets removed from $M$, then afterwards 
$I_{t} \cap X \neq \emptyset$. Thus, the only way to have $I_{t} \cap B \neq \emptyset$ yet $I_{t} \cap X=\emptyset$ is if $I_{t}$ gets added to $M$ but never removed.

Assume in addition that $I_{t} \cap A=\emptyset$. Then by compactness for all but finitely many $s$ with $\bar{I}_{s} \subseteq I_{t}$ we find that $I_{s} \subseteq U_{s}$. As we have already assumed that $I_{t} \cap X=\emptyset$, we see that these $I_{s}$ would trigger Step (3) that will remove $I_{t}$ from $M$, which contradicts our assumptions, unless they are all captured by Step (1). This in turn requires that $I_{t} \subseteq \bigcup_{s \in \mathbb{N}} B_{s}^{C}$.

Since we did reach Step (5), it must hold that $I_{t} \nsubseteq B_{t}^{C}$. Decisions justified by Step (1) do not change $B_{s}^{C}$. Therefor, there has to be some $s>t$ such that $I_{s} \cap I_{t} \cap B_{s} \neq \emptyset$. For such an $s$ we stipulate that $I_{s} \cap B=\emptyset$. But this choice can only happen in Step (4); hence, $I_{s}$ would trigger Step (3) instead, and cause the removal of $I_{t}$ from $M$. This contradicts the assumption.

Claim: If $I_{t} \cap(A \cup X) \neq \emptyset$, we decide that $I_{t}$ does intersect $B$.

If $I_{t} \cap A \neq \emptyset$, we can never apply a step leading to the decision that $I_{t} \cap B=\emptyset$, hence we will decide that $I_{t} \cap B \neq \emptyset$. Assume there is some $x \in I_{t} \cap X$, and that $I_{t}$ is the first interval for which we decide that $I_{t}$ does not intersect $B$. Due to the choice of points to add to $X$ in Step (3), we cannot have added $x$ to $X$ after having decided that $I_{t}$ does not intersect $B$. If we decided on $I_{t}$ after adding $x$ to $X$, then Step (1) cannot apply, since if $x \in I_{t} \subseteq B_{t}^{C}$, then $I_{t}$ would not have been the first interval in contradiction to our assumption. Hence Step (2) applies, and we decide that $I_{t}$ does indeed intersect $B$.

However, in some situations overtness also can be obtained for free in the support. We shall call a measure $\nu$ on a suitable metric space $C$-concentrated if for any dyadic open ball $B=B(x, r)$ either $\nu(B)>C r^{2}$ or $\nu(B)=0$ for a constant $C>0$. Define the map Concentrate $: \subseteq \mathcal{M}(\mathbf{X}) \rightrightarrows \mathcal{M}(\mathbf{X}) \times \mathbb{N}$ by $\mu \in \operatorname{dom}$ (Concentrate) iff $\mu$ is non-atomic and non-zero. Then let $(\nu, k) \in \operatorname{Concentrate}(\mu)$ if $\forall U \in \mathcal{O}(\mathbf{X}) \quad \nu(U) \leq \mu(U), \nu$ is nonatomic, non-zero and $2^{-k}$-concentrated. Let ConcentratedSupport $: \subseteq \mathcal{M}(\mathbf{X}) \times \mathbb{R} \times \mathbb{N} \rightarrow$ $\mathcal{A}(\mathbf{X}) \wedge \mathcal{V}(\mathbf{X})$ be defined via $(\nu, M, k) \in \operatorname{dom}$ (ConcentratedSupport) iff $\nu$ is $2^{-k}$ concentrated and $\nu(\mathbf{X})=M$, and ConcentratedSupport $(\nu, k)=\operatorname{supp}(\nu)$.

Proposition 43 Set $\mathbf{X}:=[0,1]$. Then Concentrate is computable.

Proof Let $\mu$ be the input. We search for some $k \in \mathbb{N}$, such that $\mu([0,1]) \geq 2^{-k}$. Now we can construct a flow $f$ from $\mu$ such that $f(\varepsilon)=2^{-k}$ and $f(v) \leq \mu\left(\mathfrak{D}_{v}^{\circ}\right)$ for any $v \in\{0,1\}^{*}$. From this flow we obtain a concentrated flow by Proposition 40, which then yields the desired concentrated measure. 
Proposition 44 Let $\mathbf{X}$ be a computable metric space. Then ConcentratedSupport is computable.

Proof The $\mathcal{V}$-name is computed as in Theorem 31. It remains to show that we can semidecide $x \notin \operatorname{supp}(\nu)$ given $M$ and $k$. For this, we test for any closed ball $\bar{B}\left(x, 2^{-l}\right)$ whether $\nu\left(\bar{B}\left(x, 2^{-l}\right)^{C}\right)>M-2^{-k-2 l}$, and answer yes if we found such a $l$. This procedure is computable.

It remains to argue that this procedure indeed semidecides $x \notin \operatorname{supp}(\nu)$. If $x \notin$ $\operatorname{supp}(\nu)$, then there is some open ball $B\left(x, 2^{-l+1}\right)$ with $B\left(x, 2^{-l+1}\right) \cap \operatorname{supp}(\nu)=\emptyset$, ie $\nu\left(B\left(x, 2^{-l+1}\right)\right)=0$. But then also $\nu\left(\bar{B}\left(x, 2^{-l}\right)\right)=0$ and thus $\nu\left(\bar{B}\left(x, 2^{-l}\right)^{C}\right)=M>$ $M-2^{-k-2 l}$. Conversely, if $\nu\left(\bar{B}\left(x, 2^{-l}\right)^{C}\right)>M-2^{-k-2 l}$, then $\nu\left(B\left(x, 2^{-l}\right)\right)<2^{-k-2 l}$. But then by definition of $\nu$ being $2^{-k}$-concentrated, already $\nu\left(B\left(x, 2^{-l}\right)\right)=0$, ie $x \notin$ $\operatorname{supp}(\nu)$.

Corollary 45 Given a non-atomic probability measure $\mu$ on $[0,1]$, we can compute a set $A \in \mathcal{V}([0,1]) \wedge \mathcal{A}([0,1])$ such that $A \subseteq \operatorname{supp}(\mu)$ and $\mu(A) \geq \frac{1}{2}$.

Proof Using the construction in the proof of Proposition 43 (and in there, Proposition 40), we obtain from $\mu$ a measure $\nu$ such that $\nu([0,1])=0.5, \nu(U) \leq \mu(U)$ for any set $U \in \mathcal{O}([0,1])$, and $\nu$ is $2^{-1}$-concentrated. By Proposition 44 we can compute $A=\operatorname{supp}(\nu) \in \mathcal{A}([0,1]) \wedge \mathcal{V}([0,1])$. By construction, $\operatorname{supp}(\nu) \subseteq \operatorname{supp}(\mu)$, and $0.5=\nu([0,1])=\nu(\operatorname{supp}(\nu)) \leq \mu(\operatorname{supp}(\nu))$.

\subsection{Some Weihrauch degrees related to Hausdorff dimension}

As mentioned in Subsection 2.3, it is often possible to calibrate closed choice principles to the strength of a particular theorem by restricting them to a (related) class of closed sets. As such, it seems reasonable to investigate closed choice for subsets of $[0,1]$ with positive Hausdorff dimension (or even Hausdorff dimension 1). A straight-forward yet important consequence of Shmerkin's construction (Theorem 27) is that this does not yield any new Weihrauch degrees:

Definition 46 Let $\mathrm{HC}_{[0,1]}$ be the restriction of $\mathrm{C}_{[0,1]}$ to $\left\{A \in \mathcal{A}([0,1]) \mid \operatorname{dim}_{\mathcal{H}}(A)>\right.$ $0\}$. Let $\mathrm{H}_{1} \mathrm{C}_{[0,1]}$ be the restriction of $\mathrm{C}_{[0,1]}$ to $\left\{A \in \mathcal{A}([0,1]) \mid \operatorname{dim}_{\mathcal{H}}(A)=1\right\}$.

Corollary $47 \quad \mathrm{HC}_{[0,1]} \equiv_{\mathrm{W}} \mathrm{H}_{1} \mathrm{C}_{[0,1]} \equiv_{\mathrm{W}} \mathrm{C}_{[0,1]}$. 
Before continuing with our investigation of measures, we shall classify the Hausdorff dimension itself. We also consider the same map, but with a different type as $\operatorname{dim}_{\mathcal{H}}: \mathcal{A}([0,1]) \rightarrow \mathbb{R}_{>}$, the investigation of which was suggested to the authors by Andrej Bauer at CCC 2014. Note that our result does make use of the fact that we have defined Hausdorff dimension only for subsets of $[0,1]$; the result generalizes directly to compact subsets of a metric space though.

Theorem $48 \operatorname{dim}_{\mathcal{H}} \equiv_{\mathrm{W}} \lim \star \lim$ and $\operatorname{dim}_{\mathcal{H}} \equiv_{\mathrm{W}} \lim$.

For the proof, we first need a couple of lemmata.

Lemma $49(d, A) \mapsto\left\{\sum_{i \in \mathbb{N}} r_{i}^{d} \mid \exists\left(x_{i}\right)_{i \in \mathbb{N}} A \subseteq \bigcup_{i \in \mathbb{N}} B\left(x_{i}, r_{i}\right)\right\}:[0,1] \times \mathcal{A}([0,1]) \rightarrow$ $\mathcal{O}\left(\mathbb{R}_{<}\right)$is computable.

Proof Given $d \in \mathbb{R}$ and $A \in \mathcal{K}([0,1])$ (using that $[0,1]$ is compact), we can compute $\left\{\left(\left(r_{0}, r_{1}, \ldots\right),\left(x_{0}, x_{1}, \ldots\right)\right) \mid A \subseteq \bigcup_{i \in \mathbb{N}} B\left(x_{i}, r_{i}\right)\right\} \in \mathcal{O}\left([0,1]^{\mathbb{N}} \times[0,1]^{\mathbb{N}}\right)$. As $[0,1]^{\mathbb{N}}$ is computably overt, we can move to $\left\{\left(r_{0}, r_{1}, \ldots\right) \mid \exists\left(x_{0}, x_{1}, \ldots\right) A \subseteq \bigcup_{i \in \mathbb{N}} B\left(x_{i}, r_{i}\right)\right\} \in$ $\mathcal{O}\left([0,1]^{\mathbb{N}}\right)$. For $d>0$, pointwise exponentiation is an open map, thus we compute $\left\{\left(r_{0}^{d}, r_{1}^{d}, \ldots\right) \mid \exists\left(x_{0}, x_{1}, \ldots\right) A \subseteq \bigcup_{i \in \mathbb{N}} B\left(x_{i}, r_{i}\right)\right\} \in \mathcal{O}\left([0,1]^{\mathbb{N}}\right)$. Then we use Lemma 6 to obtain $\left\{\sum_{i \in \mathbb{N}} r_{i}^{d} \mid \exists\left(x_{i}\right)_{i \in \mathbb{N}} A \subseteq \bigcup_{i \in \mathbb{N}} B\left(x_{i}, r_{i}\right)\right\} \in \mathcal{O}\left(\mathbb{R}_{<}\right)$.

Lemma $50\left(U_{(\cdot)} \mapsto \inf \left\{d \in[0,1] \mid \inf U_{d} \leq 0\right\}: \mathcal{C}\left([0,1], \mathcal{O}\left(\mathbb{R}_{<}\right)\right) \rightarrow \mathbb{R}_{>}\right) \leq_{\mathrm{W}} \lim$

Proof We find that inf $U_{d} \leq 0$ iff $\forall k \in \mathbb{N} 2^{-k} \in U_{d}$. We can compute $U_{d} \mapsto$ $\left\{k \in \mathbb{N} \mid 2^{-k} \in U_{d}\right\}: \mathcal{O}\left(\mathbb{R}_{<}\right) \rightarrow \mathcal{O}(\mathbb{N})$. Now (isFinite $\left.: \mathcal{O}(\mathbb{N}) \rightarrow \mathbb{S}\right) \leq_{\mathrm{W}} \lim$, as follows from arguments in Neumann and Pauly [34]. Since $\widehat{\lim } \equiv_{\mathrm{W}} \lim$, we can semidecide whether inf $U_{d}>0$ for all rational $d$ in parallel, and this suffices to obtain $\inf \left\{d \in[0,1] \mid \inf U_{d} \leq 0\right\} \in \mathbb{R}_{>}$.

A standard argument establishes:

Lemma $51\left(\left(q_{i}\right)_{i \in \mathbb{N}} \mapsto \sup _{j \in \mathbb{N}} \inf _{k \in \mathbb{N}} q_{\langle j, k\rangle}: \subseteq \mathcal{C}(\mathbb{N}, \mathbb{Q}) \rightarrow \mathbb{R}\right) \equiv_{\mathrm{W}} \lim \star \lim$.

Proof For the $\leq_{\mathrm{W}}$-direction, note that inf $\equiv_{\mathrm{W}} \sup \equiv_{\mathrm{W}} \lim \equiv_{\mathrm{W}} \widehat{\lim }$, eg v Stein [46] and Brattka and Gherardi [6].

For the $\geq$ w-direction, we employ the function $F_{2}:\{0,1\}^{\mathbb{N}} \rightarrow\{0,1\}^{\mathbb{N}}$ defined via $F_{2}(x)(n)=0$ iff $\forall i \exists k \quad x(\langle n, i, k\rangle)=0$ and $F_{2}(x)(n)=1$ otherwise. This function was 
studied in Stein [46] and Brattka [3] $]^{5}$, and in Brattka, Gherardi and Marcone [8] it is shown that $F_{2} \equiv \mathrm{w} \lim \star \lim$.

Given $x \in\{0,1\}^{\mathbb{N}}$, let $q_{\langle\langle n, j\rangle, k\rangle}:=\sum_{l \leq n} 2 \cdot 3^{-l} x(\langle l, j, k\rangle)$. Then

$$
\sup _{j} \inf _{k} q_{\langle\langle n, j\rangle, k\rangle}=\sum_{\{l \leq n \mid \neg \forall j \exists k x(\langle l, j, k\rangle)=0\}} 2 \cdot 3^{-l}
$$

and subsequently:

$$
\sup _{\langle n, j\rangle} \inf _{k} q_{\langle\langle n, j\rangle, k\rangle}=\sum_{\{l \in \mathbb{N} \mid \neg \forall j \exists k x(\langle l, j, k\rangle)=0\}} 2 \cdot 3^{-l}
$$

From such a member of the usual ternary Cantor set, the corresponding point in $\{0,1\}^{\mathbb{N}}$ can be computed. This point then is $F_{2}(x)$.

Given some closed set $A \in \mathcal{A}([0,1])$ and some proper interval $[a, b]$, let $A^{\rightarrow[a, b]} \subseteq[a, b]$ be the rescaling of $A$ into $[a, b]$, ie defined via $x \in A \Leftrightarrow a+x(b-a) \in A^{\rightarrow[a, b]}$. Not only is this a computable operation, but even $\left(A_{i}\right)_{i \in \mathbb{N}} \mapsto\{0\} \cup \bigcup_{i \in \mathbb{N}} A_{i} \rightarrow^{\left[2^{-2 i-2}, 2^{-2 i-1}\right]}$ : $\mathcal{C}(\mathbb{N}, \mathcal{A}([0,1])) \rightarrow \mathcal{A}([0,1])$ is computable. Moreover, we have:

Lemma $52 \operatorname{dim}_{\mathcal{H}}(A)=\operatorname{dim}_{\mathcal{H}}\left(A^{\rightarrow[a, b]}\right)$ and

$$
\sup _{i \in \mathbb{N}} \operatorname{dim}_{\mathcal{H}}\left(A_{i}\right)=\operatorname{dim}_{\mathcal{H}}\left(\{0\} \cup \bigcup_{i \in \mathbb{N}} A_{i}^{\rightarrow\left[2^{-2 i-2}, 2^{-2 i-1}\right]}\right)
$$

Proof of Theorem 48 We shall first prove $\operatorname{dim}_{\mathcal{H}}^{>} \leq_{\mathrm{w}} \lim$. Given some $A \in \mathcal{A}([0,1])$, we can curry the computable map from Lemma 49 to obtain:

$$
\left(d \mapsto\left\{\sum_{i \in \mathbb{N}} r_{i}^{d} \mid \exists\left(x_{i}\right)_{i \in \mathbb{N}} A \subseteq \bigcup_{i \in \mathbb{N}} B\left(x_{i}, r_{i}\right)\right\}\right) \in \mathcal{C}\left([0,1], \mathcal{O}\left(\mathbb{R}_{<}\right)\right)
$$

Using the first characterization of Hausdorff dimension in Definition 24, we see that applying the map from Lemma 49 to this just provides $\operatorname{dim}_{\mathcal{H}}^{>}(A)$. As $\left(\mathrm{id}: \mathbb{R}_{>} \rightarrow \mathbb{R}\right) \equiv_{\mathrm{W}}$ $\lim$, it immediately follows that $\operatorname{dim}_{\mathcal{H}} \leq \mathrm{w} \lim \star \lim$.

For the converse direction, we use the construction of sets of given Hausdorff dimension in Corollary 26. First, given some sequence $\left(x_{i}\right)_{i \in \mathbb{N}} \in[0,1]^{\mathbb{N}}$ we can compute a sequence of sets set $\left(A_{i}\right)_{i \in \mathbb{N}} \in \mathcal{A}([0,1])^{\mathbb{N}}$ with $\operatorname{dim}_{\mathcal{H}}\left(A_{i}\right)=x_{i}$. By Lemma 52, we

\footnotetext{
${ }^{5}$ In the previous literature, the function $F_{2}$ was denoted by $C_{2}$. We avoid this notation in order to prevent confusion with choice for the discrete two element space. Instead, our notation is inspired by de Holanda Cunha Nobrega [24].
} 
then can compute a set $A \in \mathcal{A}([0,1])$ with $\operatorname{dim}_{\mathcal{H}}(A)=\sup _{i \in \mathbb{N}} x_{i}$. Altogether, this shows $\left(\sup :[0,1]^{\mathbb{N}} \rightarrow \mathbb{R}_{>}\right) \leq_{\mathrm{W}} \operatorname{dim}_{\mathcal{H}}$. As sup : $[0,1]^{\mathbb{N}} \rightarrow \mathbb{R}_{<}$is computable, and $\mathbb{R}_{<} \wedge \mathbb{R}_{>}$is computably isomorphic to $\mathbb{R}$, this establishes $\lim \leq_{\mathrm{w}} \operatorname{dim}_{\mathcal{H}}^{>}$.

To see that $\lim \star \lim \leq_{\mathrm{W}} \operatorname{dim}_{\mathcal{H}}$, note that given $x_{i, j} \in([0,1] \cap \mathbb{Q})^{\mathbb{N} \times \mathbb{N}}$, we can compute a set $A \in \mathcal{A}([0,1])$ with $\operatorname{dim}_{\mathcal{H}}(A)=\sup _{i \in \mathbb{N}} \inf _{j \in \mathbb{N}} x_{i, j}$ by using first the construction in Subsection 2.4 and then Lemma 52. Lemma 51 shows that this suffices for the claim.

Corollary 53 There is a computable closed and computable overt set $A \in \mathcal{A}([0,1])$, such that $\operatorname{dim}_{\mathcal{H}}(A)$ is Turing-equivalent to the iterated Halting problem $\emptyset^{\prime \prime}$.

Proof Combine Theorem 48, Proposition 42 and the basic facts that isolated points do not change the Hausdorff dimension, and that any map Weihrauch equivalent to $\lim \star \lim$ maps a computable input to a solution Turing-equivalent to $\emptyset^{\prime \prime}$.

\subsection{The Frostman Lemma}

We now finally direct our attention to the maps Frost and StrictFrost introduced in Definition 30. One can prove the Frostman lemma via the min-cut/max-flow theorem, and the construction of the flows involved (if done in the right way) yields Weihrauch reductions to NonZeroFlow and MaxFlow respectively.

Lemma 54 Frost $\leq_{\mathrm{w}}$ NonZeroFlow and StrictFrost $\leq_{\mathrm{w}}$ MaxFlow.

Proof We adapt the proof of the Frostman lemma given in Fouché, Mukeru and Davie [17, Proposition 2.3], which in turn is based on the central idea to invoke the min-cut/max-flow theorem present eg in Mörters and Peres [31].

Given a closed set $A \in \mathcal{A}([0,1])$ and some $s \in[0,1]$ we construct an assignment $g:\{0,1\}^{*} \rightarrow \mathbb{R}_{0}^{+}$. As in Subsection 4.1, we assume a canonic notation $\left(\mathfrak{D}_{v}\right)_{v \in\{0,1\}^{*}}$ of the closed dyadic rational intervals in $[0,1]$. As $[0,1]$ is compact, we can semidecide if $A \cap \mathfrak{D}_{v}=\emptyset$, and we run all these tests simultaneously. When it comes to assigning a weight to $v \in\{0,1\}^{*}$, we choose $g(v):=2^{-s|v|}$ if $\mathfrak{D}_{v} \cap A=\emptyset$ has not yet been ascertained yet. If we have proof that $\mathfrak{D}_{v}$ is disjoint from $A$, we assign $g(v):=0$.

As shown eg in [17, Proposition 2.3], there is a non-zero flow compatible with $g$. (Any cut through this tree gives an upper bound for the $s$-dimensional Hausdorff content of $A$, and so the min-cut/max-flow theorem implies that there is a non-zero flow compatible with the tree.) 
Any such flow then induces a measure using the construction from Subsection 4.1. The construction ensures that this is an $s$-Frostman measure supported on $A$. By considering the local versions of the argument that there is a non-zero flow compatible with $g$, it even follows that a maximal flow compatible with $g$ yields a solution to StrictFrost.

The converse directions are obtained from the following in conjunction with preceding results:

Lemma $55 \mathrm{C}_{\mathbb{N}} \times \mathrm{H}_{1} \mathrm{C}_{[0,1]} \leq{ }_{\mathrm{W}}$ Frost.

Proof As before, we use the computable rescaling operation $B^{\rightarrow[a, b]}$. Given a nonempty closed set $A \in \mathcal{A}(\mathbb{N})$ and another closed subset $B \in \mathcal{A}([0,1])$ with $\operatorname{dim}_{\mathcal{H}}(B)=1$, we may compute the set $C:=\{0\} \cup \bigcup_{i \in A} B^{\rightarrow\left[2^{-2 i-2}, 2^{-2 i-1}\right]} \in \mathcal{A}([0,1])$, and note that this set again has Hausdorff dimension 1.

Moreover, we make use of $\mathrm{C}_{\mathbb{N}} \equiv_{\mathrm{W}} \mathrm{UC}_{\mathbb{N}}$, ie for closed choice on $\mathbb{N}$ we may safely assume that the closed set $A$ is a singleton. We invoke $\operatorname{Frost}(C, 0.5)$ to obtain some Frostman measure $\mu$, and then Corollary 32 to find some point $x$ in the support $\mu$. $A$ being a singleton ensures that 0 is isolated in $C$, hence $0 \notin \operatorname{supp}(\mu)$. But then we can compute from $x$ some $n$ with $x \in\left[2^{-2 n-2}, 2^{-2 n-1}\right]$, which answers $\mathrm{UC}_{\mathbb{N}}$, and then scale $x$ back to $[0,1]$ to obtain some point $y \in B$.

Lemma $56 \quad($ id $: \mathcal{A}(\mathbb{N}) \rightarrow \mathcal{V}(\mathbb{N})) \leq_{\mathrm{w}}$ StrictFrost.

Proof Given $A \in \mathcal{A}(\mathbb{N})$, we can compute $\left(\{0\} \cup \bigcup_{n \in A}\left[2^{-2 n-2}, 2^{-2 n-1}\right]\right)$. We use StrictFrost to find a measure with this set as support, and then Theorem 31 to obtain the set as overt set. To complete the reduction, note $n \in A \Leftrightarrow\left(2^{-2 n-2}, 2^{-2 n-1}\right) \cap$ $\left(\{0\} \cup \bigcup_{i \in A}\left[2^{-2 i-2}, 2^{-2 i-1}\right]\right) \neq \emptyset$.

Corollary $57 \mathrm{C}_{\mathbb{N}} \times \mathrm{C}_{[0,1]} \equiv_{\mathrm{W}}$ NonZeroFlow $\equiv_{\mathrm{W}}$ Frost.

Proof This is obtained by combining Lemma 54, Lemma 55, Theorem 39 and Corollary 47.

Corollary $58 \lim \equiv_{\mathrm{W}}$ MaxFlow $\equiv_{\mathrm{W}}$ StrictFrost.

Proof This is obtained by combining Lemma 54, Lemma 56, Theorem 37 and von Stein's Theorem 13. 
The first equivalence in both Corollary 57 and Corollary 58 was already known to Brattka.

A direct consequence of Corollary 57 is that a computable closed set with Hausdorff dimension $>2^{-n}$ may still fail to admit a computable $2^{-n}$-Frostman measure. Proposition 42 then shows that requiring the set to be a computable closed and overt set does not change this. On the other hand, in [17] a construction of computable Frostman measures on very special sets is given. We can make the relevant properties of the sets explicit in the following:

Lemma 59 Given $s \in[0,1]$, a set $A \in \mathcal{A}([0,1]) \wedge \mathcal{V}([0,1])$ and $p \in \mathbb{N}^{\mathbb{N}}$ such that for any dyadic interval $I$ we find $I \cap A=\emptyset$ or $A \cap I$ admits an $s$-Frostman measure $\mu$ with $\mu(I \cap A) \geq 2^{-p(-\log |I|)}$ we can compute an $s$-Frostman measure on $A$.

By Propositions 43, 44 the converse is true, too:

Corollary 60 Let $A$ admit a computable $s$-Frostman measure. Then there is a computable $B \in \mathcal{A}([0,1]) \wedge \mathcal{V}([0,1])$ with $B \subseteq A$ and a computable sequence $p \in \mathbb{N}^{\mathbb{N}}$ such that for any dyadic interval $I$ we find $I \cap A=\emptyset$ or $A \cap I$ admits an $s$-Frostman measure $\mu$ with $\mu(I \cap A) \geq 2^{-p(-\log |I|)}$. Moreover, if an $s$-Frostman measure $\nu$ on $A$ is given, we can effectively find $B$ and $p$.

\subsection{On computably universally measure 0 sets}

Recall that a set $A$ is called universally measure $O$ if there is no non-atomic non-zero Radon measure supported by $A$. Clearly every countable set is universally measure 0 . There are universally measure 0 sets with cardinality $2^{\aleph_{0}}$, however, such sets cannot be Borel. An example on the real line was constructed by Sierpiński and SzpilrajnMarczeswski [45]. Later, Zindulka even found an example of a universally measure 0 set with positive Hausdorff dimension [50]; thus, the requirement of compactness of $A$ in the Frostman lemma cannot be completely relaxed.

Our results show that in the computable world, the picture is very different: Let us call a set $A$ computably universally measure 0 if there is no non-atomic non-zero computable Radon measure supported by $A$.

Proposition 61 There is a computable closed and computable overt set with Hausdorff dimension 1 that is computably universally measure 0 . 
Proof As a consequence of Corollary 47 there is computable closed set $A \subseteq[0,1]$ without any computable points and Hausdorff-dimension 1. By Proposition 42 there is a computable closed and computable overt set $B \supseteq A$ such that the perfect core of $A$ and $B$ are the same. Assume for the sake of contradiction that $B$ admits a non-atomic non-zero computable Radon measure $\mu$ supported by $B$. As no isolated point of $B$ can be part of the support of $\mu$, we find that $\mu$ is already supported by $A$. By Corollary 32, we can compute a point $x \in \operatorname{supp}(\mu) \subseteq A$, which contradicts the choice of $A$. Thus $B$ satisfies the claim.

A much stronger effective notion is arithmetically universally measure 0 ; a set is this if it does not support any non-atomic non-zero Radon measure computable relative to some arithmetic degree. This notion (under a different moniker) was employed by Gregoriades in [20] in order to study computable Polish spaces up to $\Delta_{1}^{1}$-isomorphisms: A Polish space $\mathbf{X}$ is $\Delta_{1}^{1}$-isomorphic to $\mathbb{N}^{\mathbb{N}}$ iff it is not arithmetically universally measure 0 . Gregoriades then constructed computable closed and computable overt sets with cardinality $2^{\aleph_{0}}$ that are arithmetically universally measure 0 .

\section{References}

[1] V Brattka, Recursive characterization of computable real-valued functions and relations, Theoretical Computer Science 162 (1996) 45-77; doi: 10.1016/03043975(95)00249-9

[2] V Brattka, Computable Invariance, Theoretical Computer Science 210 (1999) 3-20; doi: 10.1016/S0304-3975(98)00095-4

[3] V Brattka, Effective Borel measurability and reducibility of functions, Mathematical Logic Quarterly 51 (2005) 19-44; doi: 10.1002/malq.200310125

[4] V Brattka, M de Brecht, A Pauly, Closed Choice and a Uniform Low Basis Theorem, Annals of Pure and Applied Logic 163 (2012) 968-1008; doi: 10.1016/j.apal.2011.12.020

[5] V Brattka, G Gherardi, Effective Choice and Boundedness Principles in Computable Analysis, Bulletin of Symbolic Logic 17 (2011) 73 - 117; arXiv:0905.4685; doi: $10.2178 / \mathrm{bsl} / 1294186663$

[6] V Brattka, G Gherardi, Weihrauch Degrees, Omniscience Principles and Weak Computability, Journal of Symbolic Logic 76 (2011) 143 - 176; arXiv:0905.4679

[7] V Brattka, G Gherardi, R Hölzl, Probabilistic computability and choice, Information and Computation 242 (2015) 249 - 286; arXiv:1312.7305; doi: 10.1016/j.ic.2015.03.005

[8] V Brattka, G Gherardi, A Marcone, The Bolzano-Weierstrass Theorem is the Jump of Weak König's Lemma, Annals of Pure and Applied Logic 163 (2012) 623-625; doi: 10.1016/j.apal.2011.10.006 
[9] V Brattka, S Le Roux, A Pauly, Connected Choice and Brouwer's Fixed Point Theorem (2012); arXiv:1206.4809

[10] V Brattka, S Le Roux, A Pauly, On the Computational Content of the Brouwer Fixed Point Theorem, from: "How the World Computes", (S Cooper, A Dawar, B Löwe, editors), Lecture Notes in Computer Science 7318, Springer Berlin Heidelberg (2012) 56-67; doi: 10.1007/978-3-642-30870-3_7

[11] V Brattka, A Pauly, Computation with Advice, Electronic Proceedings in Theoretical Computer Science 24 (2010) CCA 2010; arXiv:1006.0551

[12] V Brattka, A Pauly, On the algebraic structure of Weihrauch degrees (2016); arXiv: 1604.08348

[13] V Brattka, G Presser, Computability on subsets of metric spaces, Theoretical Computer Science 305 (2003) 43 - 76; doi: 10.1016/S0304-3975(02)00693-X

[14] P Collins, Computable Stochastic Processes (2014); arXiv:1409.4667

[15] M Escardó, Synthetic topology of datatypes and classical spaces, Electronic Notes in Theoretical Computer Science 87 (2004)

[16] K Falconer, The Geometry of Fractal Sets, Cambridge Tracts in Mathematics 85, Cambridge University Press (1986)

[17] W L Fouché, S Mukeru, G Davie, Fourier spectra of measures associated with algorithmically random Brownian motion, Logical Methods of Computer Science 10 (2014) 1-24; doi: 10.2168/LMCS-10(3:20)2014

[18] O Frostman, Potentiel d'équilibre et capacité des ensembles avec quelques applications à la théorie des fonctions, Mat. sem., Meddel. Lunds. Univ. (1935)

[19] G Gherardi, A Marcone, How incomputable is the separable Hahn-Banach theorem?, Notre Dame Journal of Formal Logic 50 (2009) 393-425; doi: 10.1215/00294527-2009018

[20] V Gregoriades, Classes of Polish spaces under effective Borel isomorphism, Memoirs of the American Mathematical Society (2016); doi: 10.1090/memo/1135

[21] V Gregoriades, T Kispéter, A Pauly, A comparison of concepts from computable analysis and effective descriptive set theory, Mathematical Structures in Computer Science (2016); doi: 10.1017/S0960129516000128

[22] K Higuchi, A Pauly, The degree-structure of Weihrauch-reducibility, Logical Methods in Computer Science 9 (2013); doi: 10.2168/LMCS-9(2:2)2013

[23] M L Hogarth, Does General Relativity Allow an Observer to View an Eternity in a Finite Time?, Foundations of Physics Letters 5 (1992) 173-181

[24] H de Holanda Cunha Nobrega, Game characterizations of function classes and Weihrauch degrees, M.Sc. thesis, University of Amsterdam (2013)

[25] M Hoyrup, C Rojas, Computability of probability measures and Martin-Löf randomness over metric spaces, Information and Computation 207 (2009) 
[26] M Hoyrup, C Rojas, K Weihrauch, Computability of the Radon-Nikodym Derivative, Computability 1 (2012) 3-13; doi: 10.3233/COM-2012-005

[27] C Kreitz, K Weihrauch, Theory of Representations, Theoretical Computer Science 38 (1985) 35-53

[28] S Le Roux, A Pauly, Weihrauch degrees of finding equilibria in sequential games (2014); arXiv:1407.5587

[29] S Le Roux, A Pauly, Finite choice, convex choice and finding roots, Logical Methods in Computer Science (2015); doi: 10.2168/LMCS-11(4:6)2015

[30] S Le Roux, A Pauly, Weihrauch Degrees of Finding Equilibria in Sequential Games, from: "Evolving Computability", (A Beckmann, V Mitrana, M Soskova, editors), Lecture Notes in Computer Science 9136, Springer (2015) 246-257; doi: 10.1007/9783-319-20028-6_25

[31] P Mörters, Y Peres, Brownian motion, Cambridge University Press (2010)

[32] U Mylatz, Vergleich unstetiger Funktionen in der Analysis, Diplomarbeit, Fachbereich Informatik, FernUniversität Hagen (1992)

[33] E Neumann, Computational problems in metric fixed point theory and their Weihrauch degrees, Logical Methods in Computer Science 11 (2015); doi: 10.2168/LMCS11(4:20)2015

[34] E Neumann, A Pauly, A topological view on algebraic computations models (2016); arXiv:1602.08004

[35] A Pauly, Representing Measurement Results, Journal of Universal Computer Science 15 (2009) 1280-1300

[36] A Pauly, How Incomputable is Finding Nash Equilibria?, Journal of Universal Computer Science 16 (2010) 2686-2710; doi: 10.3217/jucs-016-18-2686

[37] A Pauly, On the (semi)lattices induced by continuous reducibilities, Mathematical Logic Quarterly 56 (2010) 488-502; doi: 10.1002/malq.200910104

[38] A Pauly, On the topological aspects of the theory of represented spaces, Computability 5 (2016) 159-180; doi: 10.3233/COM-150049

[39] A Pauly, G Davie, W Fouché, Weihrauch-completeness for layerwise computability (2015); arXiv:1505.02091

[40] R Rettinger, K Weihrauch, Products of effective topological spaces and a uniformly computable Tychonoff Theorem, Logical Methods in Computer Science 9 (2013); doi: 10.2168/LMCS-9(4:14)2013

[41] M Schröder, Admissible Representations for Continuous Computations, PhD thesis, FernUniversität Hagen (2002)

[42] M Schröder, Extended admissibility, Theoretical Computer Science 284 (2002) 519538; doi: 10.1016/S0304-3975(01)00109-8 
[43] M Schröder, Admissible Representations for Probability Measures, Mathematical Logic Quarterly 53 (2007) 431-445; doi: 10.1002/malq.200710010

[44] P Shmerkin, Uncountable preimage of every point, MathOverflow (2010); url: http://mathoverflow.net/q/47633

[45] W Sierpiński, E Szpilrajn-Marczeswski, Remarque sur le problème de la measure, Fundamenta Mathematicae 32 (1932) 133-148

[46] T v Stein, Vergleich nicht konstruktiv lösbarer Probleme in der Analysis, Diplomarbeit, Fachbereich Informatik, FernUniversität Hagen (1989)

[47] K Weihrauch, Computability on computable metric spaces, Theoretical Computer Science 113 (1993) 191-210

[48] M Ziegler, Computability and continuity on the real arithmetic hierarchy and the power of type-2 nondeterminism, from: "Proceedings of CiE 2005", (B S Cooper, B Löwe, L Torenvliet, editors), LNCS 3526, Springer (2005) 562-571

[49] M Ziegler, Real Hypercomputation and Continuity, Theory of Computing Systems 41 (2007) 177 - 206; doi: 10.1007/s00224-006-1343-6

[50] O Zindulka, Universal measure zero, large Hausdorff dimension, and nearly Lipschitz maps, Fundamenta Mathematicae 218 (2012) 95-119 doi: 10.4064/fm218-2-1

\section{Acknowledgements}

We appreciate the incisive comments from the referees of this paper. Our research has been supported by the European Union grant agreement Marie Curie Actions People International Research Staff Exchange Scheme PIRSES-GA-2011- 294962 in Computable Analysis (COMPUTAL). The second author has also been supported by the National Research Foundation (NRF) of South Africa.

Computer Laboratory, University of Cambridge, United Kingdom, (Arno Pauly has since moved to the Department d'Informatique, Université Libre du Bruxelles.)

Department of Decision Sciences, School of Economic Sciences, University of South Africa

Arno.Pauly@cl.cam.ac.uk, fouchwl@unisa.ac.za

http://www.cl.cam.ac.uk/ amp66/

Received: 27 February $2015 \quad$ Revised: 6 March 2017 